\title{
Blending isogeometric analysis and local maximum entropy meshfree approximants
}

\author{
A. Rosolen ${ }^{1}$, M. Arroyo* \\ LaCàN, Universitat Politècnica de Catalunya (UPC), Barcelona 08034, Spain
}

\begin{abstract}
A B S T R A C T
We present a method to blend local maximum entropy (LME) meshfree approximants and isogeometric analysis. The coupling strategy exploits the optimization program behind LME approximation, treats iso geometric and LME basis functions on an equal footing in the reproducibility constraints, but views the former as data in the constrained minimization. The resulting scheme exploits the best features and over comes the main drawbacks of each of these approximants. Indeed, it preserves the high fidelity boundary representation (exact CAD geometry) of isogeometric analysis, out of reach for bare meshfree methods, and easily handles volume discretization and unstructured grids with possibly local refinement, while maintaining the smoothness and non negativity of the basis functions. We implement the method with B Splines in two dimensions, but the procedure carries over to higher spatial dimensions or to other non negative approximants such as NURBS or subdivision schemes. The performance of the method is illus trated with the heat equation, and linear and nonlinear elasticity. The ability of the proposed method to impose directly essential boundary conditions in non convex domains, and to deal with unstructured grids and local refinement in domains of complex geometry and topology is highlighted by the numerical examples.
\end{abstract}

\section{Introduction}

Approximants selected by maximum entropy (max ent) are non negative smooth meshfree approximation schemes, optimal from an information theory viewpoint $[1,2]$. The non negativity and first order reproducing conditions endow these approximants with the structure of convex geometry [1], like linear finite ele ment, natural neighbor method [3], subdivision approximants [4], or B Spline and Non Uniform Rational B Splines (NURBS) basis functions [5]. Max ent approximants have been extended to second order [6,7], and to arbitrary order by dropping non negativity [8].

Local maximum entropy (LME) approximants allow us to flexi bly control the support of the basis functions on unstructured grids of points $[1,9]$. Their non negativity endow them with variation diminishing properties, as well as with a weak Kronecker delta property on the boundary of the convex hull of the set of nodes [1], by which interior basis functions vanish at the boundary of the convex hull, and basis functions vanish at any given face unless the corresponding node belongs to that face of the boundary. Thanks to this property, essential boundary conditions can be eas ily imposed on polygonal convex domains, in contrast with other

\footnotetext{
* Corresponding author.

E-mail address: marino.arroyo@upc.edu (M. Arroyo).
}

meshfree methods [10]. Furthermore, the evaluation of the LME basis functions is very efficient using duality methods [1]. The main drawback of these approximants is given by the inherent lim itation of meshfree methods to represent complex boundaries with high fidelity. In such methods, the boundaries that can be repre sented by a mere collection of points are polytopes, either the con vex hull or more controllable domains given by alpha shapes [11]. Furthermore, the weak Kronecker delta property of LME approxi mants does not hold in non convex parts of the domain [1].

Motivated by the recent impetus on isogeometric analysis [5,12], which aims at integrating Computer Aided Design (CAD) technologies, such as B Splines, NURBS or subdivision surfaces [4], and engineering analysis, we propose here using such high fidelity description of the boundary of the domain, while approx imating the interior with max ent methods. Remarkably, the limitations of LME approximants and of isogeometric analysis are in some sense complementary, since the main drawback of the latter is precisely the rigidity imposed by the NURBS frame work on the volume meshing, which requires special techniques to go beyond tensor product meshes and accommodate trimmed surfaces, local refinement, or incongruent surface descriptions at opposing faces. Some of these issues are partially addressed in 2D with T Spline technologies [13 17], hierarchical B Splines [18] or trimming techniques [19], but largely open in 3D $[20,21]$. Three dimensional subdivision schemes, producing 
smooth convex approximants from unstructured grids, are still the topic of current research [22].

The goal of the proposed method is to unify in a common frame work the geometric fidelity of isogeometric boundary representa tions with the flexibility of meshfree approximants in the bulk of the domain. Since both B Splines and LME approximants are con vex schemes, we will show that they can be coupled through the constraints in a max ent program. The resulting approximation scheme automatically retains the non negativity and smoothness of the B Spline and LME parents. Although max ent approximants can be extended to higher order consistency, at the expense of a more involved formulation [6,7], numerical experiments show that first order consistent approximants perform very well, even in high order partial differential equations. In [7], we showed that first order LME approximants attain the same accuracy as 5th or der B Splines for structural vibrations, and are comparable to sec ond order max ent approximation schemes in a fourth order phase field model [23], or in thin shell problems [24,25], where they also compete with subdivision finite elements.

In the same spirit of the method presented here, the NURBS en hanced finite element method (NEFEM) [26] adopts a NURBS boundary representation, coupled to standard finite elements in the interior of the domain. This approach exploits the high fidelity geometry representation of isogeometric analysis, but does not in sist in preserving the smoothness and positivity of the basis func tions, placing more emphasis in the high order reproducibility conditions. On the other hand, Moving Least Squares (MLS) mesh

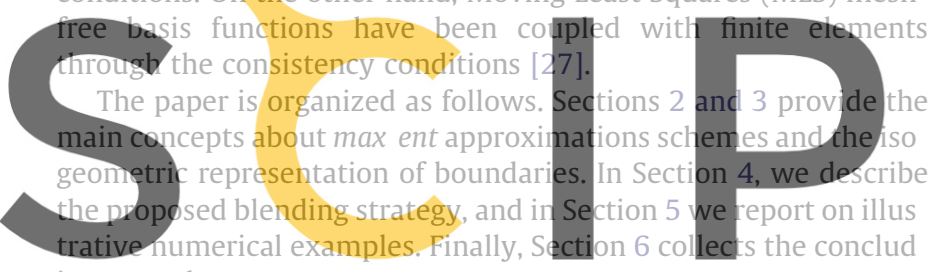
ing remarks.

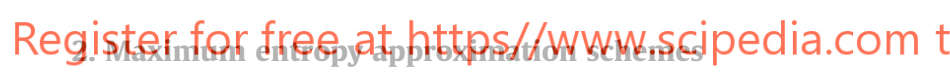 \\ In information theory and statistical inference, the principle of} max ent is a means to infer the probability distribution, which best represents the current state of knowledge about a process, consis tently with a priori information. This principle was adopted in [1,2] to generate the least biased basis functions for nodal data approx imation. The key in this information theoretical viewpoint is to interpret the approximants as probability distributions. This inter pretation follows from the partition of unity and the fact that we require the approximants to be non negative.

More concretely, consider the approximation of a function in a domain $\Omega \subset \mathbb{R}^{d}$ as a linear combination of basis functions associ ated with a set of nodes $X \quad\left\{\boldsymbol{x}_{a}\right\}_{a} 1, \ldots, N \subset \mathbb{R}^{d}$,

$u(\boldsymbol{x}) \approx u^{h}(\boldsymbol{x}) \quad \sum_{a}^{N} p_{a}(\boldsymbol{x}) u_{a}$.

Rather than defining explicitly the basis functions $p_{a}(\boldsymbol{x})$, we view them as unknowns, which need to fulfill the partition of unity $\sum_{a}^{N}{ }_{1} p_{a}(\boldsymbol{x}) \quad 1$ and the first order consistency condition $\sum_{a}^{N}{ }_{1} p_{a}(\boldsymbol{x}) \boldsymbol{x}_{a} \quad \boldsymbol{x}$. Additionally, we demand that $p_{a}(\boldsymbol{x}) \geqslant 0$. Compar ing these conditions with the definition of the convex hull of the set of nodes

$\operatorname{conv} X \quad\left\{\boldsymbol{x} \in \mathbb{R}^{d} \mid \boldsymbol{x} \quad \sum_{a}^{N} \eta_{a} \boldsymbol{x}_{a}\right.$, with $\left.\eta_{a} \geqslant 0, \sum_{a}^{N} \eta_{a} 1\right\}$,

it follows that such an approximation scheme can only be defined in domain satisfying $\Omega \subset$ convX.
If the node set is composed of more than $d+1$ affinely indepen dent points, there exist infinitely many convex approximation schemes, and the principle of max ent emerges as a selection prin ciple. These basis functions can be computed by maximizing the information entropy subject to the constraints given by the repro ducibility conditions [1,2]. The max ent framework is quite flexible and allows us to consider other related approaches. The LME approximants [1] represent the optimal compromise (in the Pareto sense) between two competing objectives: (i) maximum locality of the basis functions and (ii) maximum information entropy of the scheme.

The convex program defining the LME approximants is

$\begin{array}{rlll}(\mathrm{LME}) \text { For fixed } & \boldsymbol{x} \text { minimize } & \sum_{a 1}^{N} \beta_{a} p_{a}\left|\boldsymbol{x} \quad \boldsymbol{x}_{a}\right|^{2}+\sum_{a 1}^{N} p_{a} \ln p_{a}, \\ \text { subject to } \quad & p_{a} \geqslant 0, \quad a \quad 1, \ldots, N, \\ & \sum_{a 1}^{N} p_{a} \quad 1, \sum_{a}^{N} p_{a} \boldsymbol{x}_{a} \quad \boldsymbol{x}\end{array}$

where the non negative parameters $\beta_{a}$ weigh the relative impor tance given to each objective in each nodal position [9].

The above program is convex, smooth and feasible for any spa tial dimension $d$ (as long as $\boldsymbol{x} \in \operatorname{conv} X$ ), and produces $C^{\infty}$ meshfree non negative functions $p_{a}(\boldsymbol{x})$ [1]. Moreover, the constraints (con sistency conditions) guarantee solutions that reproduce exactly af fine functions (see $[6,7,28,29]$ for higher order approaches).

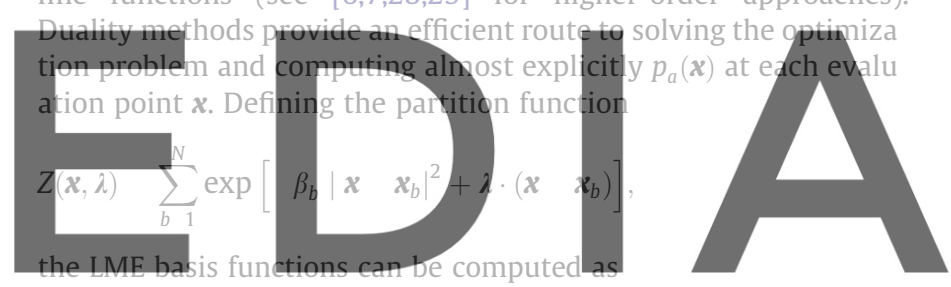

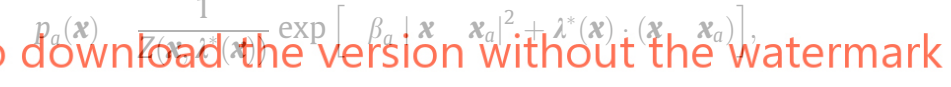

where the Lagrange multiplier for the linear consistency condition is the unique minimizer of a smooth convex unconstrained optimi zation problem, minimizing $\ln Z$, efficiently solved with Newton's method [1].

The parameters $\beta_{a} \quad \gamma_{a} / h_{a}^{2}$, where $\gamma_{a}$ is a dimensionless aspect ratio parameter and $h_{a}$ the typical nodal spacing, allow us to control the locality (the support size) of the basis functions [1,9]. The approximants become sharper and narrower as the dimensionless parameter $\gamma_{a}$ increases, and for values close to 4 and above they are nearly indistinguishable from the affine Delaunay basis functions, as illustrated in Fig. 1 in 1D. As $\gamma_{a}$ tends to infinity, it has been mathematically shown that the affine functions supported on the Delaunay triangulation of the node set are recovered [1]. In

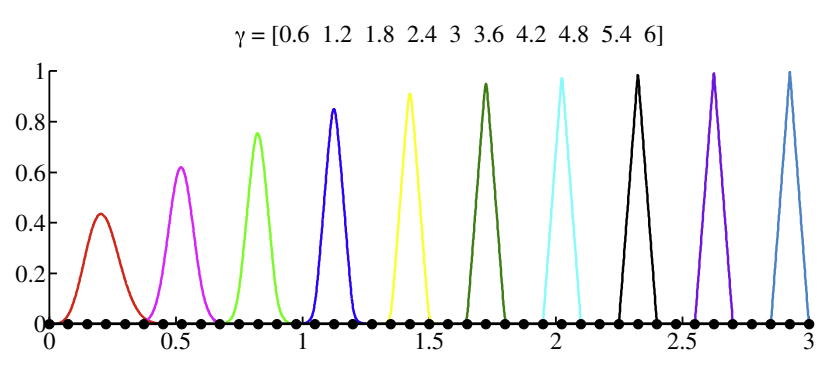

Fig. 1. LME approximants enable a seamless and smooth transition from meshfree to Delaunay affine basis functions. The transition is controlled by the nondimensional nodal parameters $\gamma_{a}$, which here take linearly varying values from 0.6 (left) to 6 (right). 


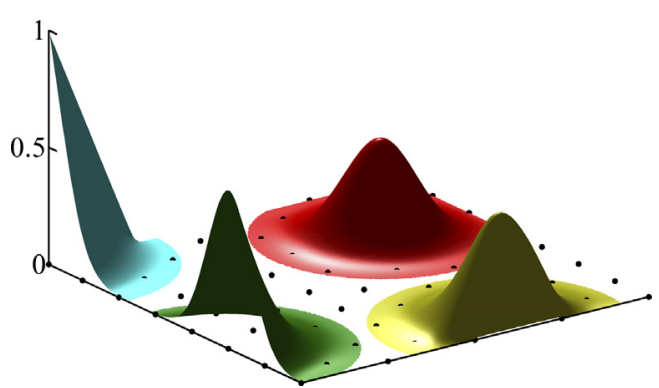

Fig. 2. Illustration of LME basis functions in a two-dimensional domain $\left(\gamma_{a}\right.$

1.6).

Fig. 2 we illustrate the LME approximants in a two dimensional domain for a parameter $\gamma_{a} \quad 1.6$.

In Fig. 3 we illustrate a LME basis function corresponding to the interior point of a non convex domain. The picture highlights the limitations of LME approximants that we are addressing in this work: (i) the inherent inability of meshfree methods to represent complex boundaries with high fidelity and (ii) the loss of the weak Kronecker delta property of LME approximants in non convex parts of the domain.

3. Isogeometric boundary representation

We here provide the minimal concepts behind the B Spline ba sis functions, curves, and surfaces, and outline the basic ideas of isogeometric analysis (see $[12,30]$ for the sake of simplicity, we stick to B Sp pling strategy is directly applicab boundary representations.

A closed B Spline curve, or a set of to NUR the proposed cou or subdivision urves defining two dimensionaldomain. In three dimensions, the boundary wil

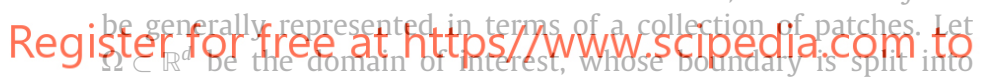
B Spline patches $\partial \Omega \cup_{\alpha} \Gamma_{\alpha}$. For $d \quad 2$, each piece of the boundary is described by a parameterized curve $\boldsymbol{C}_{\alpha}:[0,1] \mapsto \Gamma_{\alpha}$ given by

$\boldsymbol{C}_{\alpha}(\xi) \quad \sum_{i} N_{i}^{p}(\xi) \boldsymbol{P}_{i}$

where $N_{i}^{p}(\xi)$ denote the 1D B Spline basis functions of degree $p$ associated with a given knot span and $\boldsymbol{P}_{i} \in \mathbb{R}^{2}$ are the control points. For $d \quad 3$, each patch is parametrized by $\boldsymbol{S}_{\alpha}:[0,1] \times[0,1] \mapsto \Gamma_{\alpha}$ gi ven in terms of tensor product $B$ Spline functions as

$\boldsymbol{S}_{\alpha}\left(\xi_{1}, \xi_{2}\right) \quad \sum_{i} \sum_{j} N_{i}^{p}\left(\xi_{1}\right) M_{j}^{q}\left(\xi_{2}\right) \boldsymbol{P}_{i, j}$,

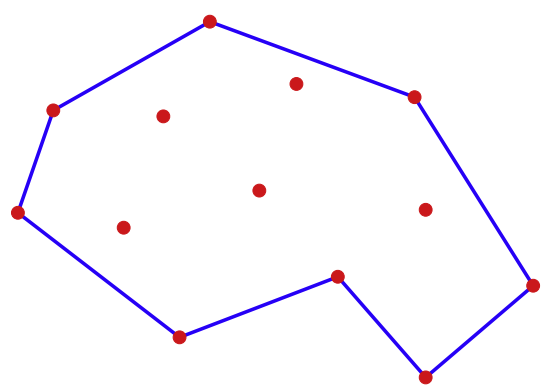

where the control points $\boldsymbol{P}_{i, j}$ are now points in three dimensions. Different continuity may be required across patches, from simple continuity of the surface $\cup_{\alpha} \Gamma_{\alpha}$ (watertight condition) to higher smoothness conditions. Here, we view the boundary representation as given and assume it is well defined.

As a prelude to the coupling strategies between isogeometric analysis and the LME approximation schemes, we introduce the isogeometric domain description. B Spline approximants are de fined in the parametric domain, while meshfree approximants are naturally defined in physical space. The coupling strategies re quire both sets of approximants to be on an equal footing. The iso geometric mapping allows us to express the B Spline approximants as functions in physical space.

To fix the ideas, consider a surface patch $\Gamma_{\alpha} \subset \partial \Omega$, and define now an associated volume patch parameterization $\boldsymbol{V}_{\alpha}\left(\xi_{1}, \xi_{2}, \xi_{3}\right):[0,1]^{3} \mapsto \Omega_{\alpha} \subset \Omega$ given by

$\boldsymbol{V}_{\alpha}\left(\xi_{1}, \xi_{2}, \xi_{3}\right) \quad \sum \sum_{j} \sum_{k} \underbrace{N_{i}^{p}\left(\xi_{1}\right) M_{j}^{q}\left(\xi_{2}\right) L_{k}^{r}\left(\xi_{3}\right)} \boldsymbol{P}_{i, j, k}$,

where the control points $\boldsymbol{P}_{i, j, k}$ need to satisfy $\boldsymbol{P}_{i, j, 1} \quad \boldsymbol{P}_{i, j}$ for the meth od to be isogeometric, i.e. $\boldsymbol{V}_{\alpha}\left(\xi_{1}, \xi_{2}, 0\right) \quad \boldsymbol{S}_{\alpha}\left(\xi_{1}, \xi_{2}\right)$. The approxi mants of isogeometric analysis, viewed as function of physical space, are simply

\section{$\widetilde{N}_{i, j, k}^{p, q, r}(\boldsymbol{x}) \quad N_{i, j, k}^{p, q, r}\left(\boldsymbol{V}_{\alpha}{ }^{1}(\boldsymbol{x})\right) \quad N_{i, j, k}^{p, q, r} \circ \boldsymbol{V}_{\alpha}{ }^{1}(\boldsymbol{x})$}

For coupling with the LME basis functions, we will only need a thin

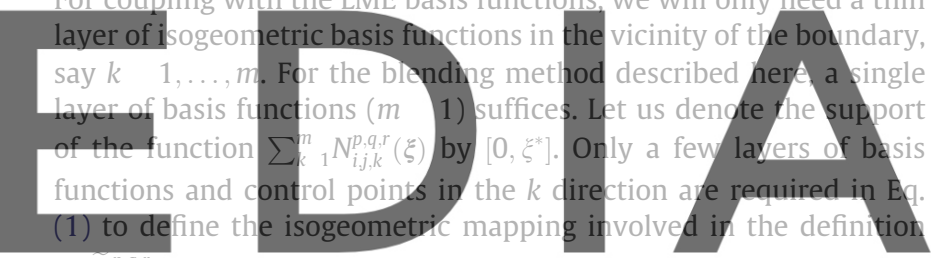
of $\bar{N}_{i, j, k}^{p, q}(\boldsymbol{x})$ for $k \quad 1, \ldots, m$. In other words, we are only interested in the mapping $V_{\alpha}$ in the domain [0.1 ${ }^{2} \times\left[0,{ }^{*}\right]$ It suffices to extend such that $\sum_{k}^{n}{ }_{1} N_{i, j, k}^{p, q}(\xi) \quad 1$ in $\left[0, \xi^{*}\right]$. For example, for cubic B Splines and one basis function in the $k$ direction ( $m$ 1), the isogeometric mapping that defines $\widetilde{N}_{i, j, 1}^{p, q, r}(\boldsymbol{x})$ requires only 4 layers of control points ( $n$ 4). See Fig. 4 for an illustration.

The proposed method only requires the volume isogeometric description in a very thin layer adhered to the boundary of the do main. Consequently, all the difficulties of isogeometric methods associated with volume meshing for general CAD descriptions (topological obstructions, incongruent surface patches, local refinement), arising from the rigidity of the tensor product struc ture, are not present here. It is however important to consistently

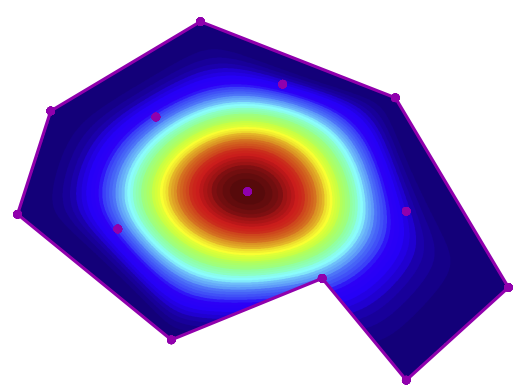

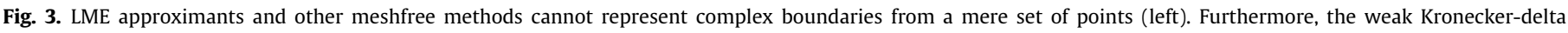

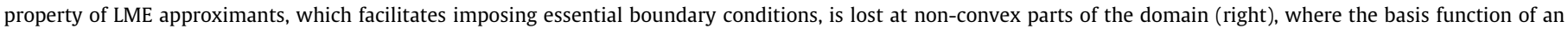
interior node does not vanish in part of the boundary. 


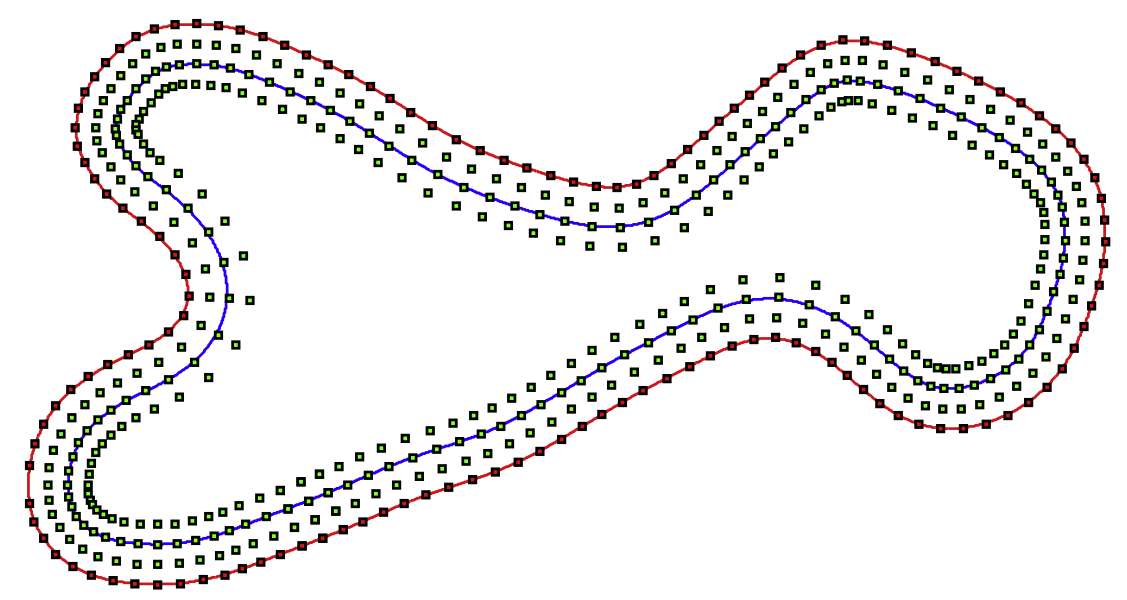

Fig. 4. Two-dimensional example of the isogeometric boundary representation. Here, a periodic B-Spline curve (red line) is described by the control points depicted by red squares. The control points involved in the volumetric isogeometric mapping defined in a narrow region next to the boundary are represented by the green squares, together with the red squares. The blue curve represents $V_{x}\left(\xi_{1}, \xi^{*}\right)$, i.e. the support of the layer of volumetric isogeometric B-Spline functions considered here, $\mathbb{N}_{i 1}^{3.3}(x)$, lies between the red and blue curves, $\boldsymbol{V}_{\alpha}\left([0,1] \times\left[0, \xi^{*}\right]\right)$. We denote and relabel these isogeometric basis functions as $N_{a}(\boldsymbol{x})$ for $a \in \mathscr{I}_{B S}$. The only purpose of the control points in green is to define the isogeometric mapping for $N_{a}(\boldsymbol{x}), a \in \mathscr{I}_{B S}$. (For interpretation of the references to colour in this figure caption, the reader is referred to the web version of this article.

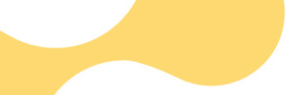

define the volume extensions $\mathbf{V}_{\alpha}$ in such a way that the paramet rizations of neighboring patches are conforming, which can be eas ily accomplished by selecting appropriately the one dimensional basis function along the $k$ direction and the control points. For this
to be possible, mild restrictions on the order of the $\mathrm{B}$ Spline
descriptions in the different patches arise.
To couple them with the LME approximants, the basis functions
and assodiated control points, $\widetilde{N}_{i, j, k}^{p, q}(\boldsymbol{x})$ and $\boldsymbol{P}_{i, j, k}$ for $k$ 1, m, are
relabeled as $N_{a}(\boldsymbol{x})$ and $\boldsymbol{x}_{a}$ for $a \in \mathscr{I}_{B S}$, where $\mathscr{I}_{B S}$ den otes the set of
global indices labeling B Spline nodes. It is impoltant to attach the control points to these basis functions since they participate in the Regist ordef concistency condition, which the isogeometric B Spline

$\sum_{i} \sum_{j} \sum_{k} \widetilde{N}_{i, j, k}^{p, q, r}(\boldsymbol{x}) \boldsymbol{P}_{i, j, k} \quad \boldsymbol{x}$.

In practice, the quadrature is performed in physical space, not in the parametric space where the B Spline functions are immediately evaluated. Therefore, the basis functions $N_{a}(\boldsymbol{x})$ for $a \in \mathscr{I}_{B S}$ need to be evaluated at arbitrary points, which involves computing $\boldsymbol{V}_{\alpha}{ }^{1}$; gi ven $\boldsymbol{x}$, we seek $\boldsymbol{\xi}$ such that $\boldsymbol{V}_{\alpha}(\boldsymbol{\xi}) \quad \boldsymbol{x}$. In practice, this can be effi ciently and robustly obtained by minimizing $f(\xi) \quad\left|\begin{array}{ll}\boldsymbol{V}_{\alpha}(\boldsymbol{\xi}) & \boldsymbol{x}\end{array}\right|^{2}$ with Newton's method and line search, and providing good initial guesses.

\section{Blending through the constraints}

We have explored three different strategies to blend LME approximants and isogeometric analysis: (i) the partition of unity method [31], (ii) the maximization of the relative entropy [29,32], and (iii) the imposition of the reproducibility conditions with a maximum entropy optimization program, and found that (iii) is the simplest and most natural from a max ent viewpoint. Consequently, henceforth we only focus in this strategy, and pro vide a brief account of the other alternatives in Appendix A.

The key idea is to consider the B Spline basis functions $N_{b}(\boldsymbol{x})$, for $b \in \mathscr{I}_{B S}$ defined in a narrow region adhered to $\partial \boldsymbol{\Omega}$, as known prob abilities (data) in the statistical inference optimization program. The integration of this information through the constraints allows us to reformulate the (LME) convex optimization problem pre sented in Section 2 as

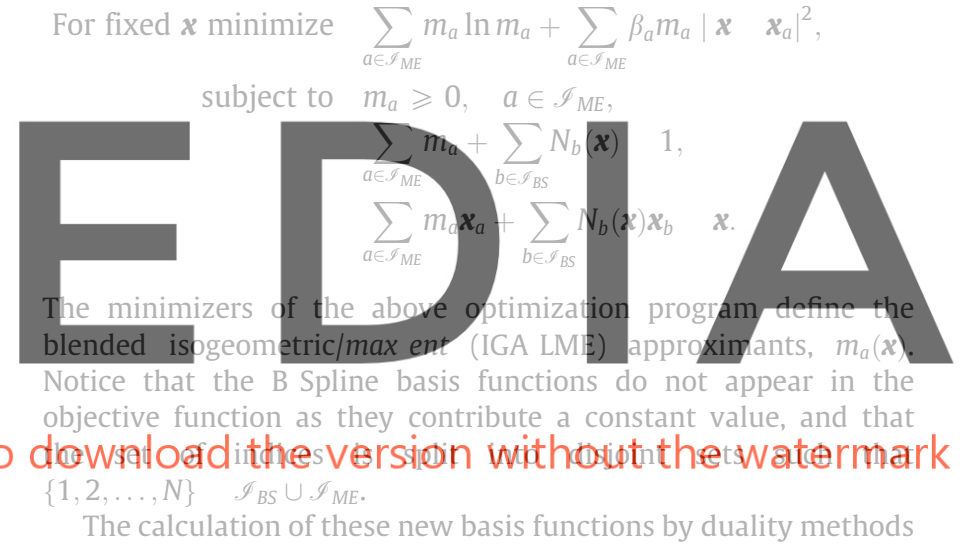

is straightforward, but it is very important to appropriateiy formu late the constraints to obtain well behaved Lagrange multipliers. Indeed, even for regular LME approximants the linear consistency

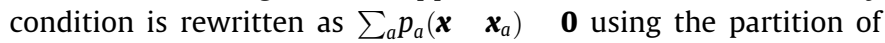
unity condition, to obtain bounded multipliers in the interior of the domain. Here, it proves sufficient to define

$A(\boldsymbol{x}) \quad 1 \sum_{b \in \mathscr{I}_{B S}} N_{b}(\boldsymbol{x})$ and $\boldsymbol{Y}(\boldsymbol{x}) \sum_{b \in \mathscr{T}_{B S}} N_{b}(\boldsymbol{x})\left(\boldsymbol{x} \quad \boldsymbol{x}_{b}\right)$,

to rewrite the first order consistency condition as

$$
\sum_{a \in \mathscr{I}_{M E}} m_{a}\left[\begin{array}{lll}
\boldsymbol{x}_{a} & \boldsymbol{x} & \frac{\boldsymbol{Y}(\boldsymbol{x})}{A(\boldsymbol{x})}
\end{array}\right] \quad \mathbf{0}
$$

at the points where $A(\boldsymbol{x})>0$. This leads to bounded Lagrange mul tipliers, which are also well behaved at the boundary of the blend ing region. Where this is not the case, the approximation is fully given by the isogeometric scheme since these non negative func tions add up to one.

The Lagrangian for the optimization program above is then

$$
\begin{aligned}
& \mathscr{L}\left(m_{a}, \lambda_{0}, \lambda\right) \sum_{a \in \mathscr{I}_{M E}} m_{a} \ln m_{a}+\sum_{a \in \mathscr{I}_{M E}} \beta_{a} m_{a}\left|\boldsymbol{x} \quad \boldsymbol{x}_{a}\right|^{2} \\
& +\lambda_{0}\left(\sum_{a \in \mathscr{I}_{M E}} m_{a} A(\boldsymbol{x})\right)+\lambda \cdot \sum_{a \in \mathscr{I}_{M E}} m_{a}\left[\begin{array}{lll}
\boldsymbol{x}_{a} & \boldsymbol{x} & \frac{\boldsymbol{Y}(\boldsymbol{x})}{A(\boldsymbol{x})}
\end{array}\right] .
\end{aligned}
$$

The stationarity conditions, together with the partition of unity con straint, lead to 
$m_{a}(\boldsymbol{x}) \quad \frac{\exp \left[f_{a}\left(\lambda^{*}(\boldsymbol{x}) ; \boldsymbol{x}\right)\right]}{Z\left(\lambda^{*}(\boldsymbol{x}) ; \boldsymbol{x}\right)}$

where

$f_{a}(\lambda ; \boldsymbol{x}) \quad \beta_{a}\left|\boldsymbol{x} \quad \boldsymbol{x}_{a}\right|^{2}+\lambda \cdot\left(\begin{array}{ll}\boldsymbol{x} & \left.\boldsymbol{x}_{a}+\frac{\boldsymbol{Y}(\boldsymbol{x})}{A(\boldsymbol{x})}\right)\end{array}\right.$

and the reduced partition function is given by

$Z(\lambda ; \boldsymbol{x}) \quad \frac{1}{A(\boldsymbol{x})} \sum_{a \in \mathscr{I}_{M E}} \exp \left[f_{a}(\lambda ; \boldsymbol{x})\right]$.

The optimal Lagrange multiplier $\lambda^{*}(\boldsymbol{x})$ can be computed by maxi mizing the reduced dual Lagrange function

$\lambda^{*}(\boldsymbol{x}) \quad \arg \max _{\lambda \in \mathbb{R}^{d}}[A(\boldsymbol{x}) \ln Z(\lambda ; \boldsymbol{x})]$.

The continuity of the resulting approximants is given by that of the $B$ Splines. The expressions for the spatial derivatives of the approx imants are provided in Appendix B.

Fig. 5 shows the IGA LME approximation schemes for a two dimensional domain described by a periodic B Spline curve (red line). The support of the B Spline basis functions $N_{a}(\boldsymbol{x})$ for $a \in \mathscr{I}_{B S}$ lies within the region between the red and the blue lines. It can be observed that the basis functions of interior nodes vanish in $\partial \Omega$, even in non convex parts, as the layer of isogeometric functions shield the IGA LME approximants from the boundary. For the blending optimization program to

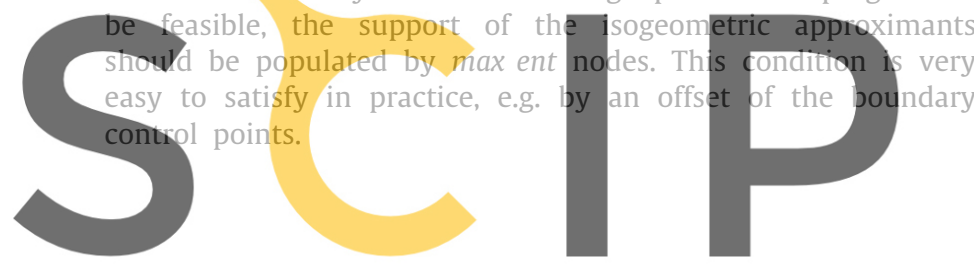

\section{Numerical examples}

We illustrate next the ability of the proposed method to handle local refinement, incongruent boundary representations, and to impose directly essential boundary conditions in non convex do mains, possibly with complex topology. For this, we consider first the heat equation, and then linear and nonlinear elasticity. Before presenting the numerical examples, we provide details on the numerical implementation.

\subsection{Implementation details}

In all the examples presented here, we adopt an aspect ratio parameter of $\gamma$ 1.6, which produces moderately spread basis functions as illustrated in Fig. 2. We describe all the boundaries with cubic B Splines, and offset a copy of the boundary control points inwards to populate the blending region, as illustrated in Fig. 5. The rest of the domain is then discretized with an unstruc tured grid generated with standard tools allowing for local refine ment [33 35].

The integration cells and quadrature points are illustrated in Fig. 6 (right). The integration points for the interior cells are gener ated with standard Gaussian quadrature rules in a background Del aunay mesh supported on the nodes. On the other hand, for the boundary cells, we use quadrature rules recently developed for high order curved elements [36] and in NEFEM applications [26]. Subdivision of the boundaries cells into smaller cells is another op

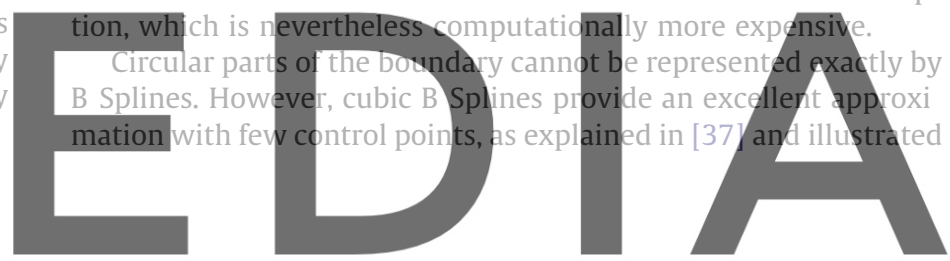

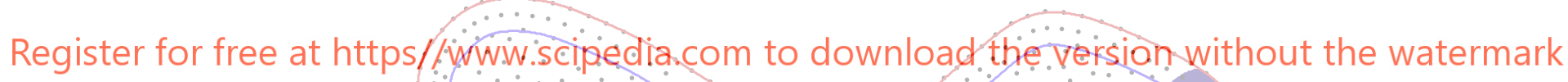
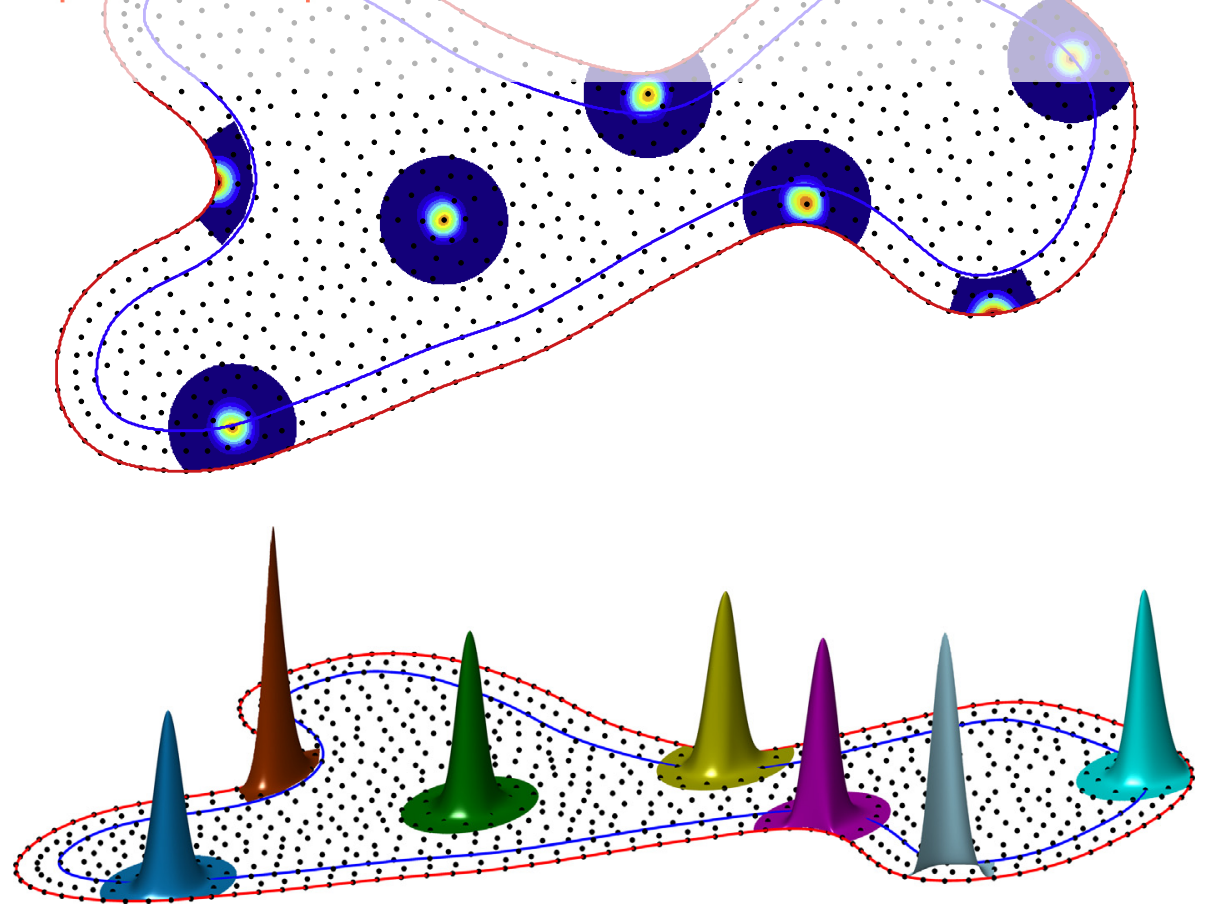

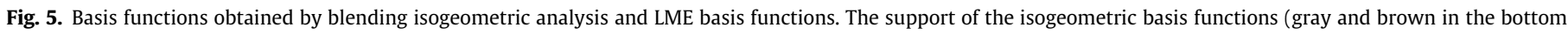

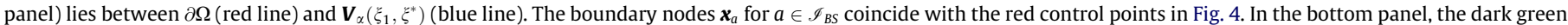

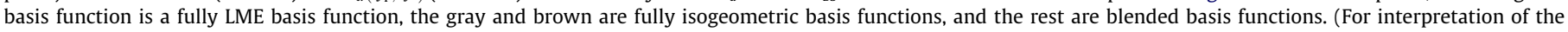
references to colour in this figure legend, the reader is referred to the web version of this article.) 

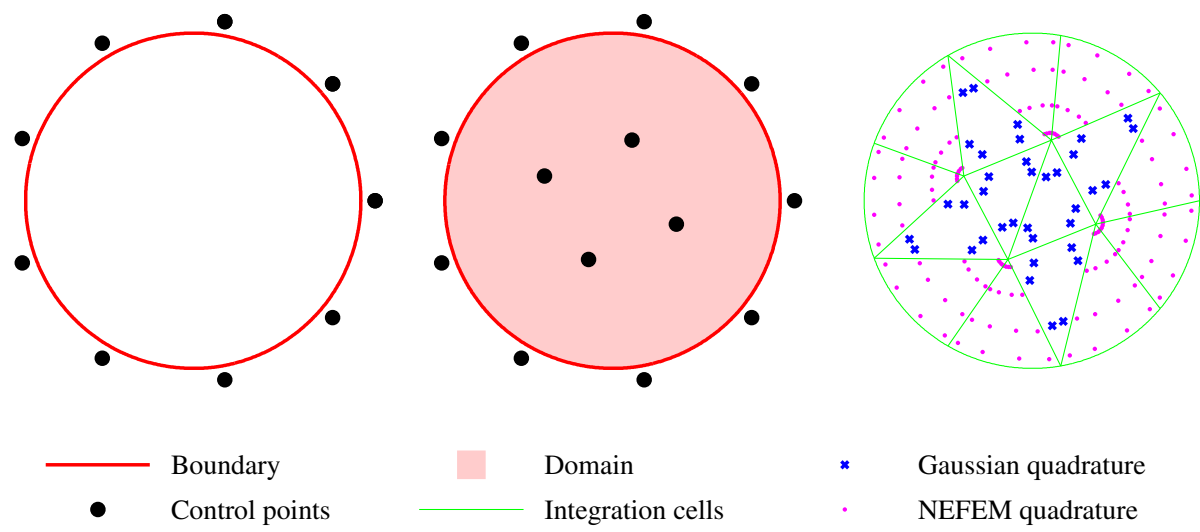

$* \quad$ Gaussian quadrature
NEFEM quadrature

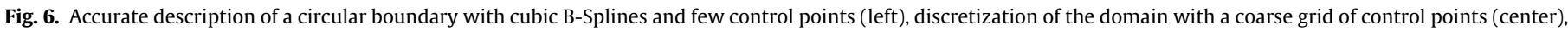
and Gaussian and NEFEM quadrature rules for interior and boundary integration cells (right).

in Fig. 6 (left). The key is to generate the coordinates of the $N_{C P}$ con trol points representing the circle with the formula $P_{i} \quad\left\{R_{p} \cos \left[\left(\begin{array}{ll}i & 1\end{array}\right) \theta\right], R_{p} \sin \left[\left(\begin{array}{ll}i & 1\end{array}\right) \theta\right]\right\}_{i}{ }_{1} N_{c p}$, where $R_{p} \frac{3}{2+\cos (\theta)} R$, $R$ is the radius of the circle, $\theta \quad \frac{2 \pi}{N_{C P} p}$, and $p \quad 3$ represents the order of the B Splines. The parametric space is given by a periodic knot vector of length $N_{k v} \quad N_{C P}+p+1$ and defined as $k v \quad\left[0 \frac{1}{N_{C P}+p} \frac{2}{N_{C P}+p} \cdots \frac{N_{C P}+p}{N_{C P}+p} 1\right]$, being the range of evaluation bounded by the knots $p+1$ and $N_{C P}$ tion to pepresent the circular boundaries appearing in the exam
ples of this section. by a periodic B Spline boundary representation (that of Fig. 5), illustrating the ability of the proposed method to impose essential

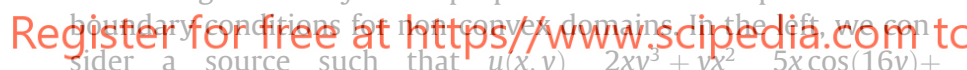
$x^{5} \cos (8 x)$ is the exact solution, and prescribe the exact essential boundary conditions. The numerical solution is highly accurate, with a relative error of 0.0023 in the $L_{2}$ norm. In the right, we illus trate a solution with homogenous Dirichlet data for the source $s(x, y) \quad 40 x^{3} \quad 8 y$. These two examples show that the proposed method allows us to impose directly essential boundary conditions in the non convex parts of the domain, circumventing a shortcom ing of LME approximation schemes [1] and meshfree methods in general [10].
Fig. 8 shows the numerical solution of the heat equation in a do main with three holes, where the exterior and the interior bound aries are represented with periodic B Spline curves. We impose constant Dirichlet data in each disjoint part of the boundary and a source. We insist that imposing Dirichlet boundary conditions is trivial for the IGA LME scheme, since only isogeometric basis functions are non zero at the boundary. This example illustrates the flexibility of the proposed approach in dealing with multiple boundary patches with different levels of refinement. A standard
isogeometric analysis of this problem would require significant
preprocessing, partitioning the domain into several two dimen
sional patches describing a logically tensor product structure, pos
sibly stitching these patches with specialized techniques to have a
globally smooth approximation, and refining some of the boundary
representations to have congruent boundary representations in a
given patch. An isogeometric analysis with local control of the refinement level would in addition require using $\mathrm{T}$ Spline or hier

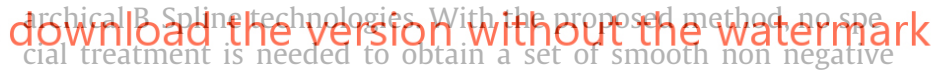
approximants with local refinement.

\subsection{Linear elasticity in a plate with a circular hole}

We solve numerically the linear elasticity boundary problem of an infinite plate with a hole subject to a far field uniaxial traction, whose exact solution is known in closed form [38]. A square com putational domain $[5,5] \times[5,5]$ with an interior circle of radius $R \quad 1$ is discretized with a uniform but unstructured distribution of
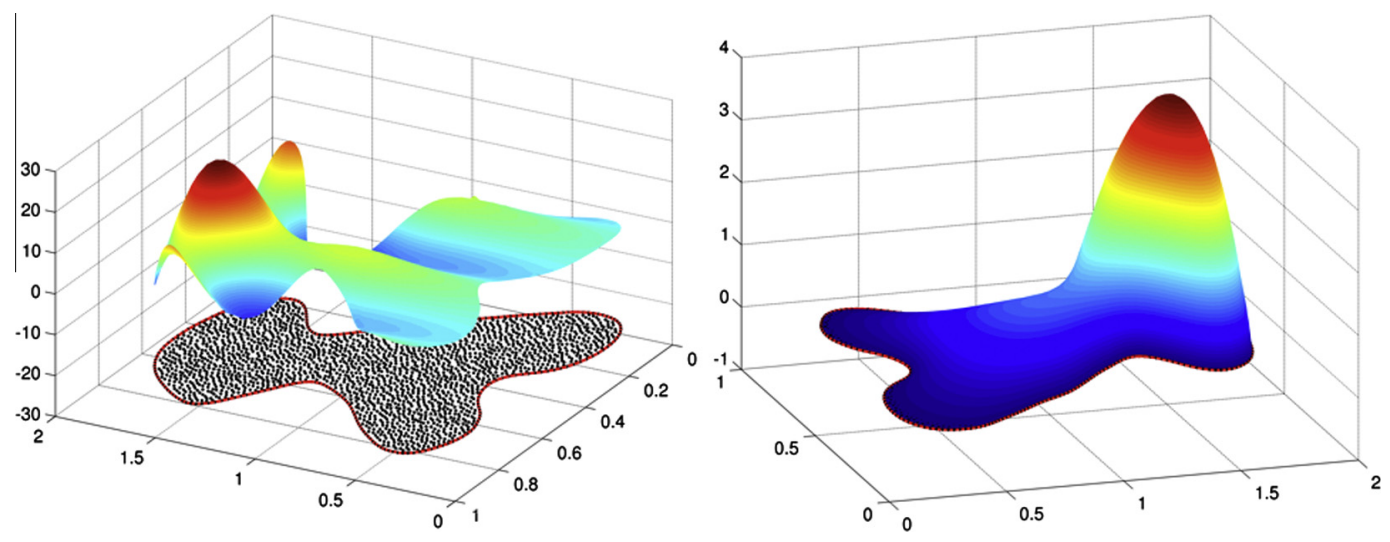

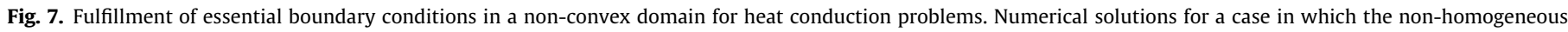
Dirichlet data are analytically prescribed (left) and for an example where homogeneous essential boundary conditions are imposed (right). 

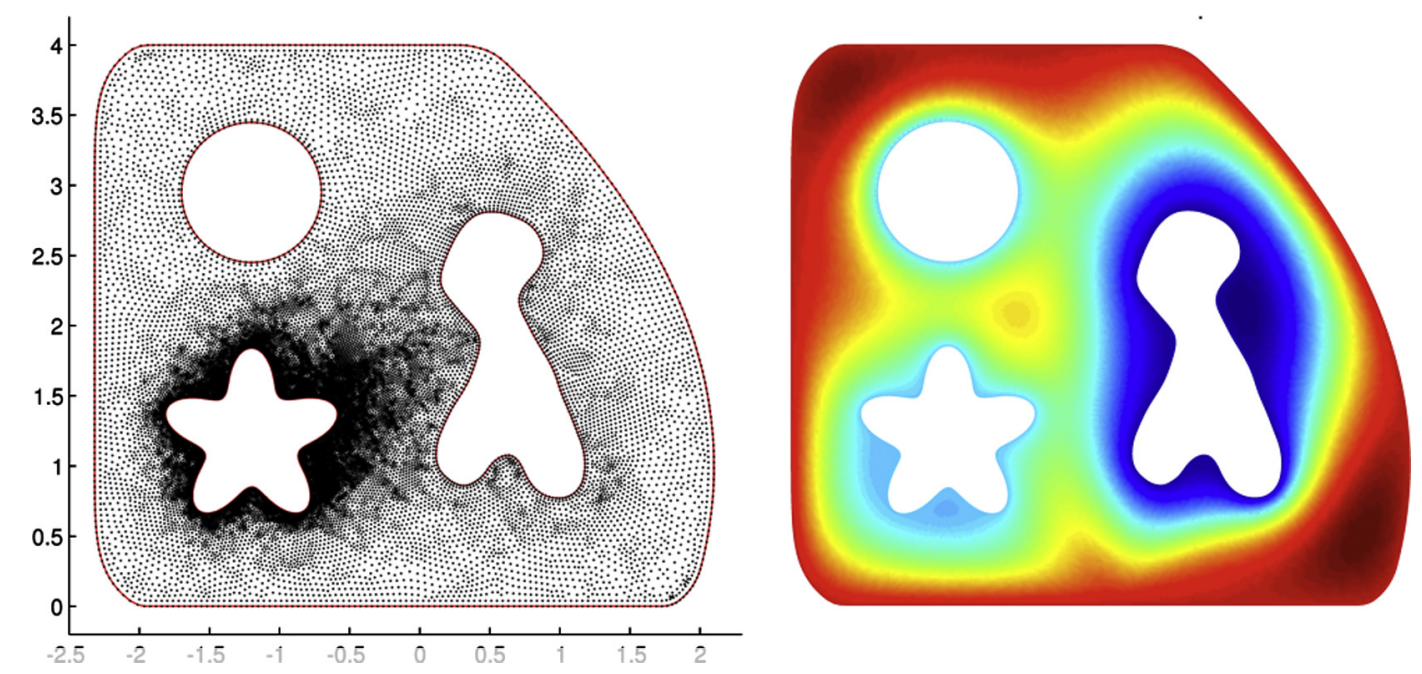

Fig. 8. Locally refined grid of control points (left) and numerical solution (right) of a Poisson problem in a domain with three holes and prescribed constant Dirichlet data on each of the disjoint sub-boundaries. The exterior and interior boundaries are described with B-Spline curves.
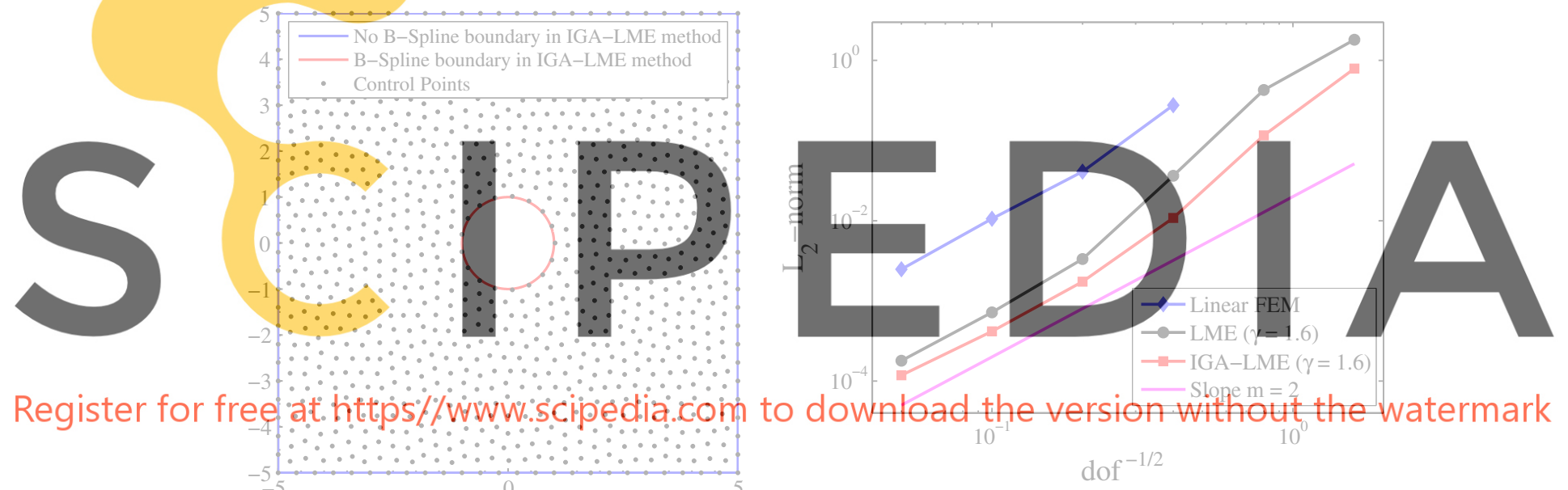

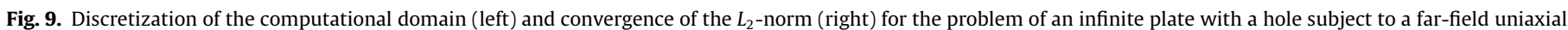
traction.

points, as illustrated in Fig. 9 (left) for a grid of 1,033 control points. The problem is solved with Neumann boundary conditions and the exact tractions. A Young's modulus of $E \quad 1$ and a Poisson's ratio of $v \quad 0.45$ are used for the numerical calculations with linear FEM, LME $(\gamma \quad 1.6)$ and IGA LME approximants.

Fig. 9 (right) shows the convergence in the $L_{2}$ norm as a func tion of the nominal grid spacing estimated as $h$ dof ${ }^{1 / 2}$ for the three numerical schemes, together with a guiding line with slope 2. The three methods converge with the optimal rate. While the
LME solution is significantly more accurate than linear finite ele ments, as previously reported, the enhancement of IGA LME with respect to LME approximants is mild but systematic.

The maximum and minimum stresses at the circumference of the hole computed with the different approximation schemes con sidered here for several levels of refinement are given in Table 1. The stresses calculated with IGA LME approximants are much more accurate than those computed with the other methods. Fig. 10 (left) shows the tangential or hoop stress $\sigma_{\theta \theta}$ at the circumference of the

Table 1

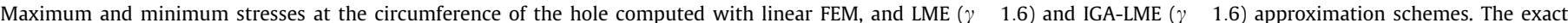
stresses are also indicated.

\begin{tabular}{|c|c|c|c|c|c|c|c|c|}
\hline \multirow[t]{2}{*}{$h$} & \multicolumn{4}{|l|}{$\sigma^{\min }$} & \multicolumn{4}{|l|}{$\sigma^{\max }$} \\
\hline & Exact & FEM & LME & IGA-LME & Exact & FEM & LME & IGA-LME \\
\hline 0.4 & -1.0 & -0.7664 & -0.8218 & -1.0722 & 3.0 & 2.9927 & 3.0776 & 3.0692 \\
\hline 0.2 & -1.0 & -0.9702 & -0.9412 & -1.0292 & 3.0 & 3.0987 & 3.0818 & 3.0176 \\
\hline 0.1 & -1.0 & -1.0368 & -0.9731 & -1.0111 & 3.0 & 3.0869 & 3.0134 & 3.0095 \\
\hline 0.05 & -1.0 & -1.0279 & -0.9811 & -1.0010 & 3.0 & 3.0843 & 3.0082 & 3.0007 \\
\hline
\end{tabular}



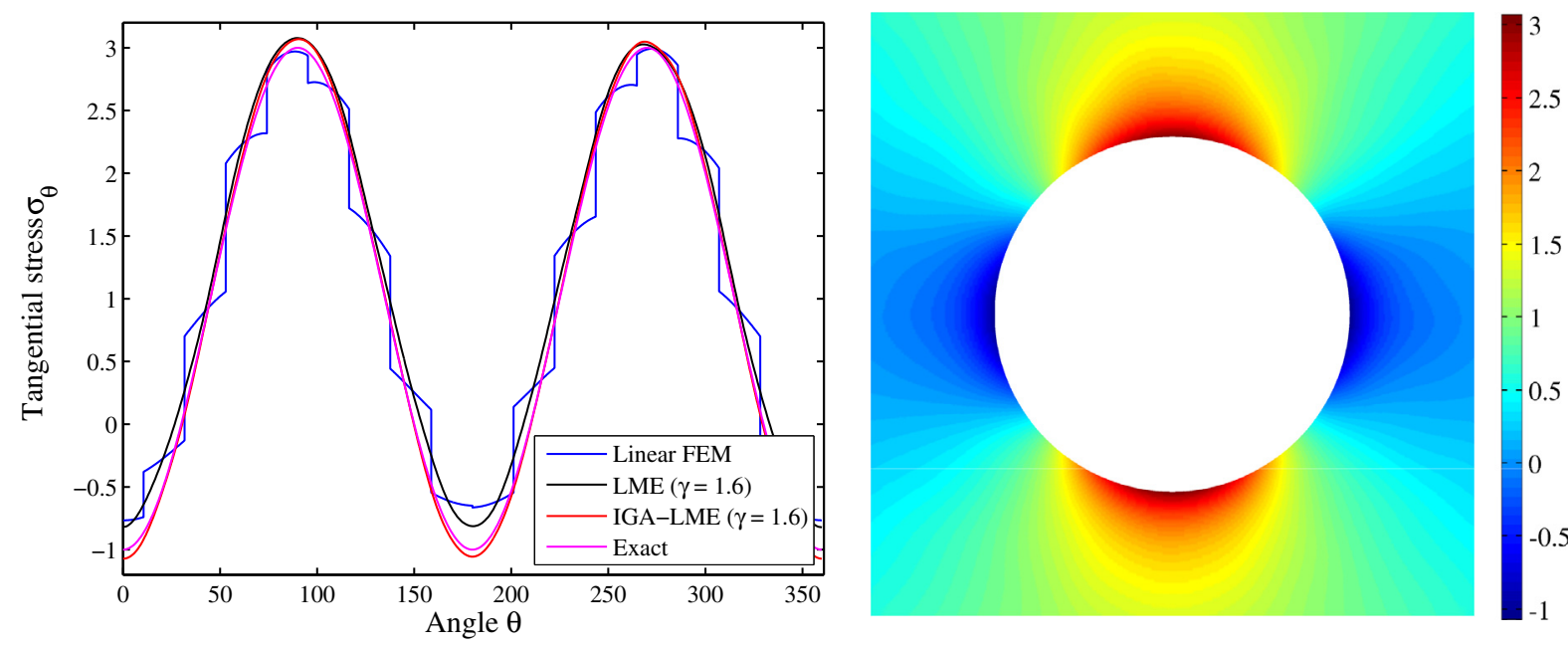

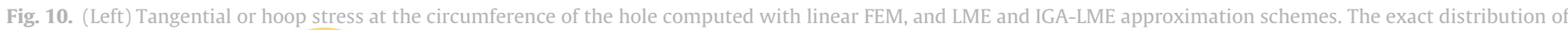

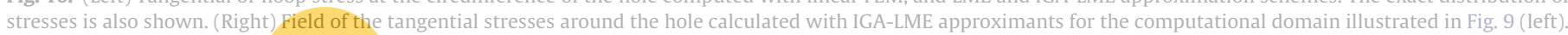

hole for the computational domain illustrated in Fig. 9 (left). The smoothness of the stresses obtained with the LME and IGA LME methods is noteworthy. The stress field around the hole calculated with IGA LME approximants is illustrated in Fig. 10 (right).

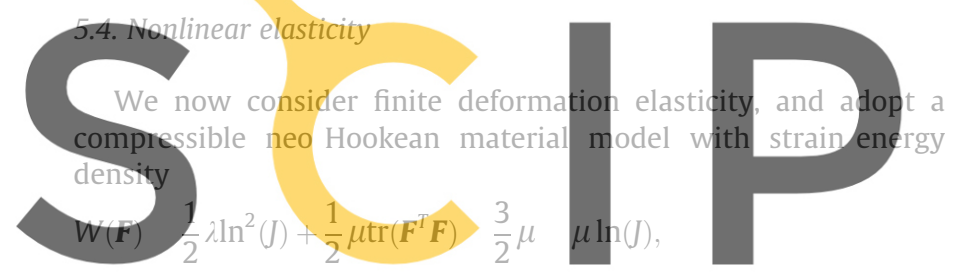

where $\boldsymbol{F}$ is the deformation gradient, $J \quad \operatorname{det}(\boldsymbol{F})$, and $\lambda$ and $\mu$ are the

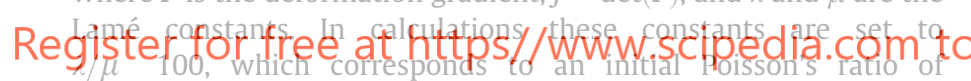
0.495 .
We first analyze a nonlinearly elastic body with a non convex domain clamped at the two interior boundaries, and progressively reduce the distance between these two holes to compress the sam ple, see Fig. 11. We describe the three boundaries with periodic B Splines and discretize the domain with an unstructured and locally

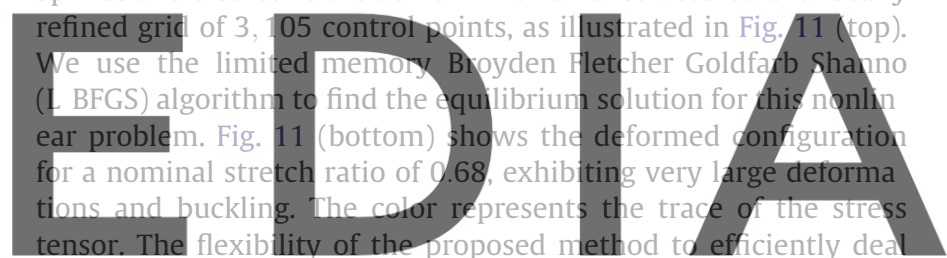
with a complex domain, retaining the high fidelity boundary representation, is noteworthy. The figure also suggests the applica

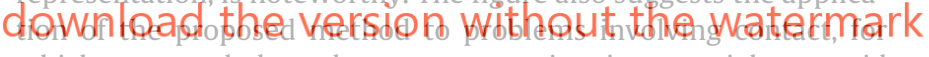
which a smooth boundary representation is essential to avoid
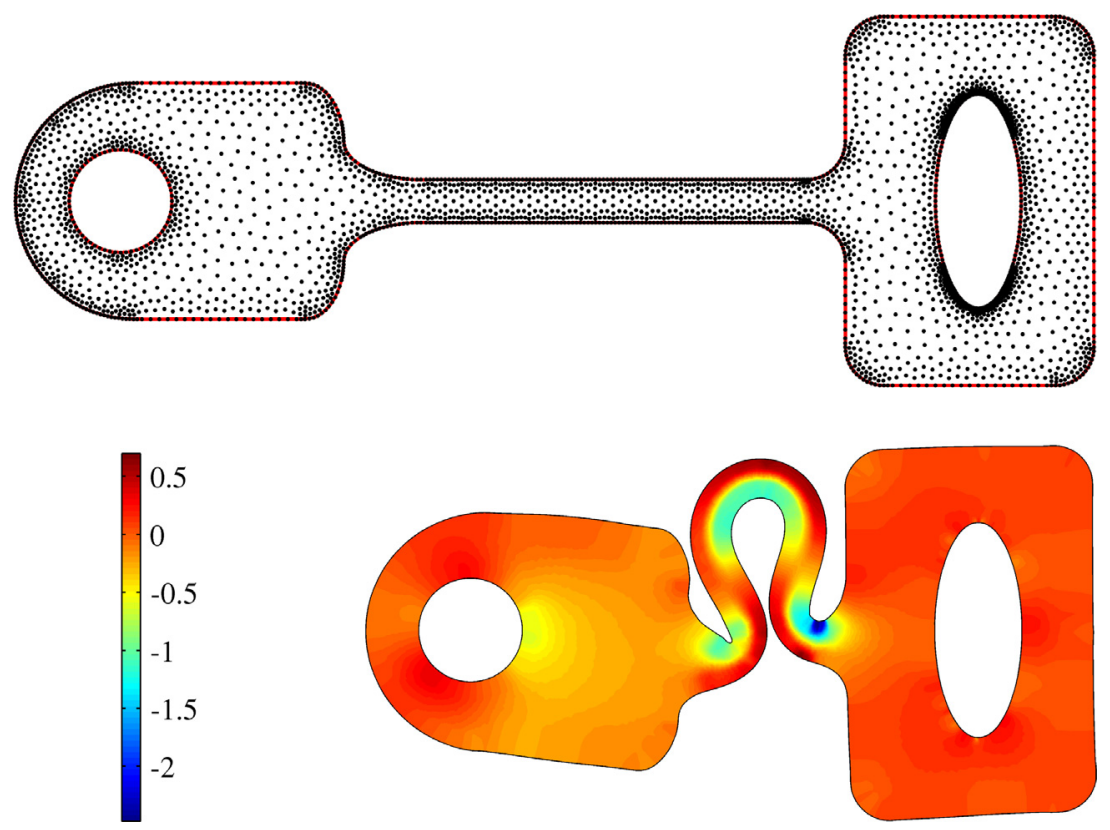

Fig. 11. Locally refined grid of control points (top) and numerical solution (bottom) of a neo-Hookean hyperelastic non-convex domain clamped on the two interior boundaries and subject to prescribed compressive deformation (the nominal stretch ratio is 0.68 ). The color represents the trace of the stress tensor. 

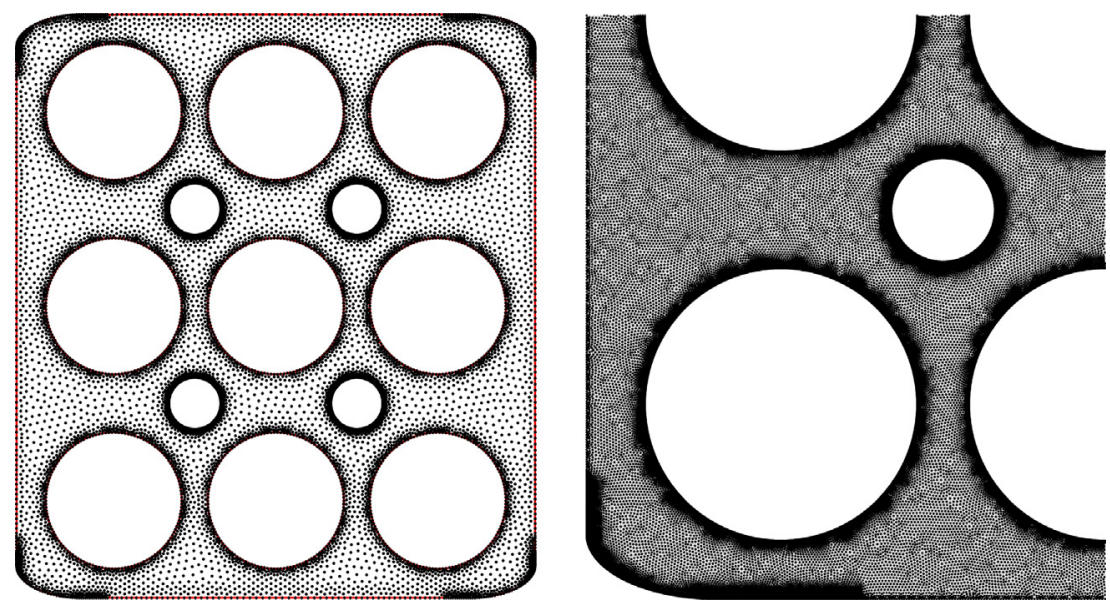

Fig. 12. IGA-LME node distribution (left) and detail of the FEM mesh (right) for a hyperelastic plate perforated with 13 circular holes.

spurious contact forces due to corners in a standard finite element mesh. This is the topic of current research.

We now consider a perforated plate (see in Fig. 12) clamped on two opposite sides, and subject to prescribed displacement. We consider the plate under compression and stretching. The exterior boundary and the 13 circular holes are described with periodic B Splines. The radii of the small and large circles are $R_{S} \quad 0.4$ and $R \quad 1.05$, and the lateral dimensions of the domain are 8 and 9.

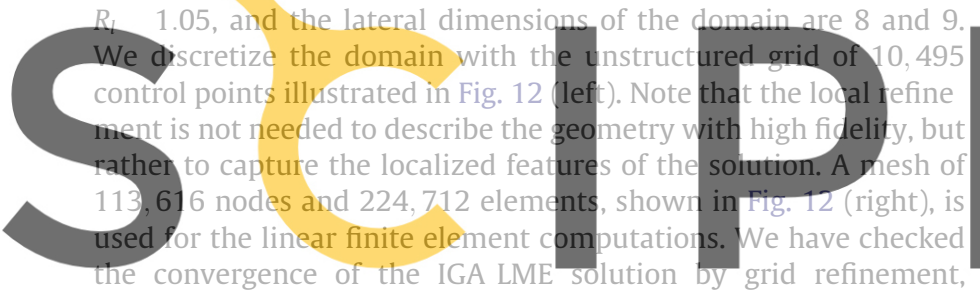
obtaining nearly identical results

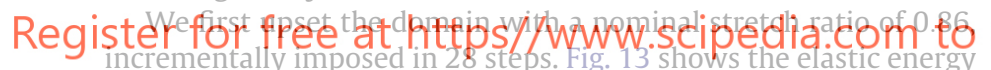
as a function of deformation, calculated here as $(\ell L) / L$, where L 8 and $\ell$ are the initial and final horizontal lengths of the plate. The buckling events at 5\%, 9\% and $11 \%$ are apparent. In Fig. 14, we illustrate the deformed configurations at the 3 post

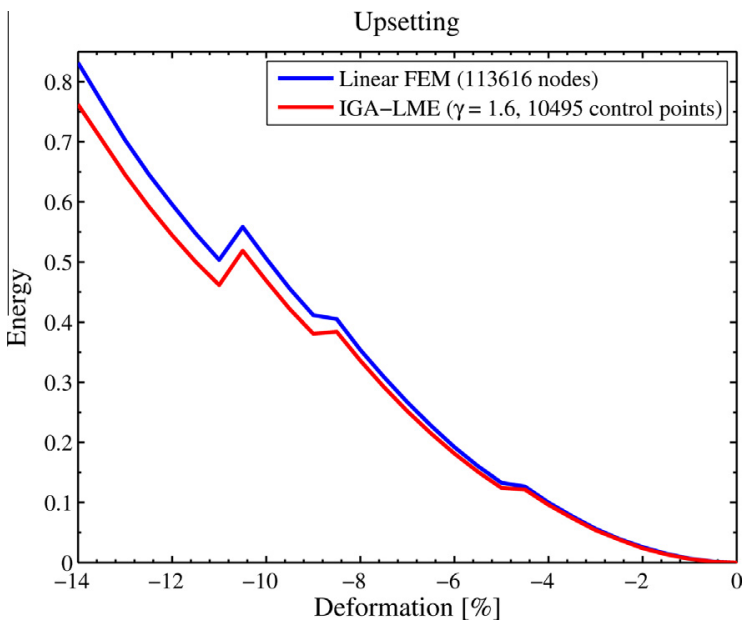

Fig. 13. Elastic energy as a function of deformation for a hyperelastic perforated plate subject to prescribed compressive deformations (the nominal stretch ratio is 0.86 ). Note the buckling events at deformations of $-5 \%,-9 \%$ and $-11 \%$, and the superior accuracy (measured as lower energy) of the IGA-LME approximants in comparison with a linear FEM model with one order of magnitude more degrees of freedom. buckling states and for the final equilibrium state. Note the ability of the IGA LME method to capture large deformations and nonlin earities. Note also the lower elastic energy (up to 9\%), indicative of a more accurate numerical solution, obtained with IGA LME as compared with linear FEM with one order of magnitude more de grees of freedom. The superior performance of the LME approxi mants as compared to linear FEM in nearly incompressible

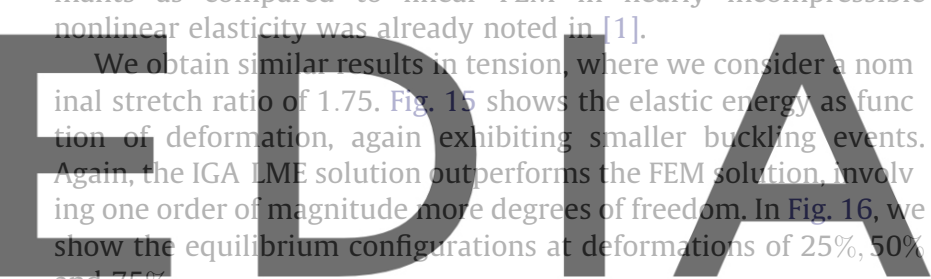

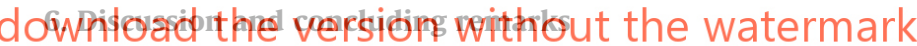

We have presented a method to produce smooth non negative approximants that describe the geometry with high fidelity (exact CAD representation) and easily handle unstructured and locally re fined volume discretizations. Although we have exercised the method in 2D here, it is readily applicable to higher dimensions. The proposed approximation schemes blend local maximum entro py approximants and isogeometric analysis through the reproduc ibility constraints in a maximum entropy convex optimization program. We have implemented the formulation with B Splines, but the method directly carries over to other non negative approx imants such as NURBS, or subdivision surfaces.

We have examined two other alternatives to blend maximum entropy approximants and isogeometric analysis, which rely on the partition of unity method, and on the maximization of the rel ative entropy with specific prior functions and constraints. Such approaches are more complex and impose stronger requirements on the node distribution, as compared to the method based on the reproducibility constraints.

The flexibility of the method for the volume discretization of domains of complex topology with isogeometric boundary fidelity, including incongruent B Spline patches and local refinement, is noteworthy, as shown with the heat equation, and linear and non linear elasticity problems. We have illustrated the ability of the IGA LME approximants to impose directly essential boundary con ditions in non convex domains.

The numerical examples presented here highlight the flexibil ity of the method, but do not exhibit a large sensitivity on the geometry representation. Other applications such as electromag 

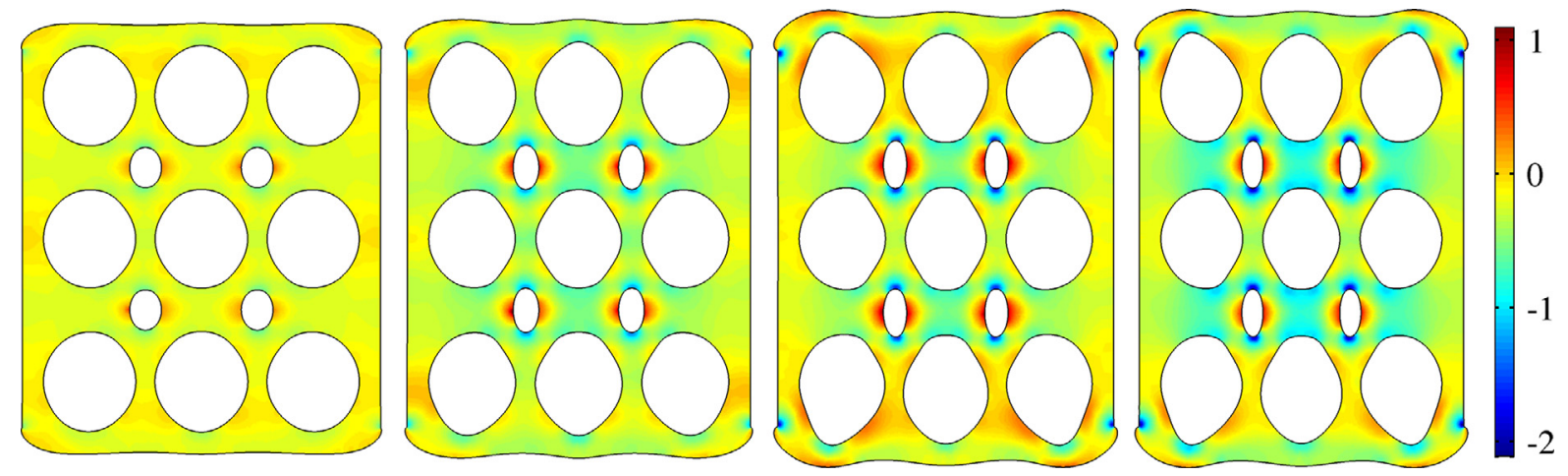

Fig. 14. Post-buckling and final deformed configurations for a hyperelastic perforated plate subject to prescribed compressive deformations of $-5 \%,-9 \%,-11 \%$ and $-14 \%$. The color represents the trace of the stress tensor.
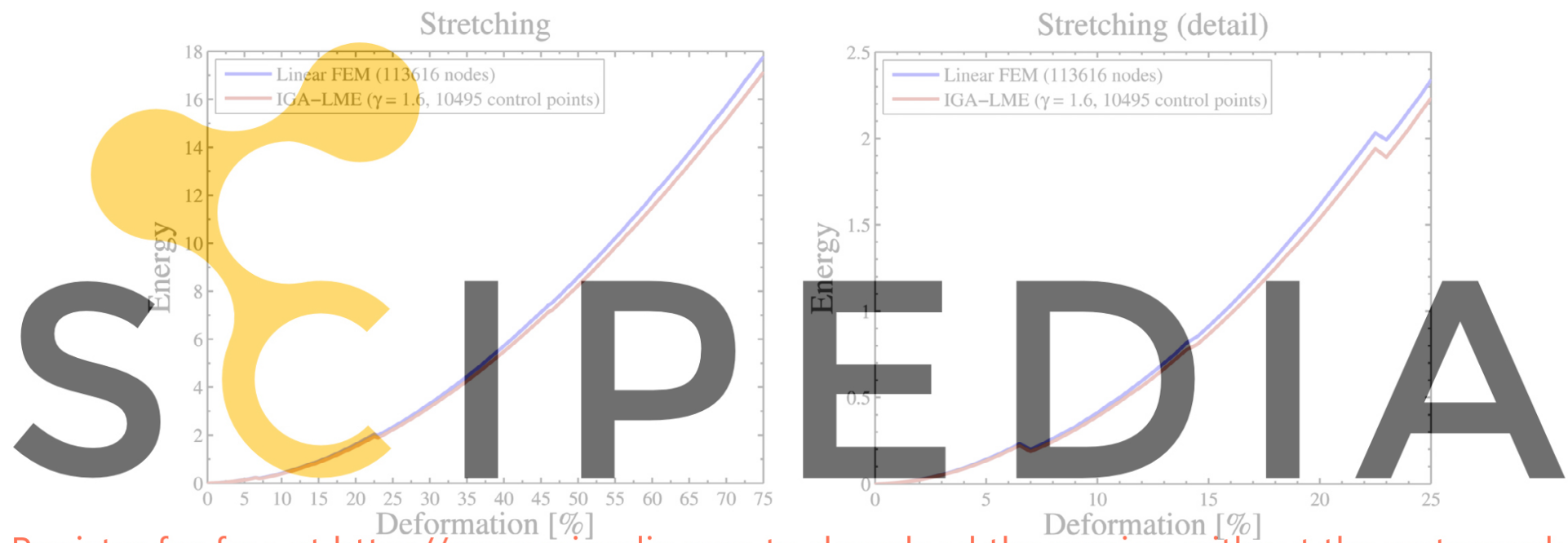

Register for free at https//www.scipedia.com to download the version without the watermark

Fig. 15. Energy as a function of deformation for a hyperelastic perforated plate subject to prescribed extensional deformations (the nominal stretch ratio is 1.75 ).
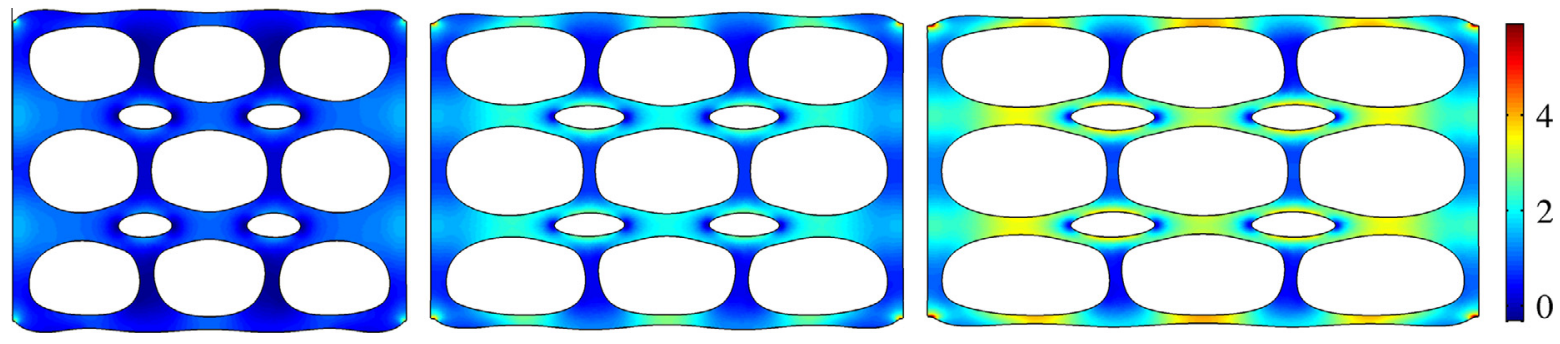

Fig. 16. Deformed configurations for a hyperelastic perforated plate subject to prescribed extensional deformations of $25 \%, 50 \%$ and $75 \%$. The color represents the trace of the stress tensor.

netic scattering, flows around airfoils and blunt bodies, nanopho tonics, or contact problems, can further benefit from the proposed method. The approximants can also be combined with a point set manifold processing methodology [24] to describe the boundaries of thin shells with high fidelity. On the other hand, the application of the proposed method to industrial problems requires a system atic treatment of bodies defined by multiple patches in 3D, and a streamlined integration with commercial CAD systems, which do not seem to present important obstacles. We finally note that, in stead of considering a thin crust of isogeometric basis functions adhered to the boundary, it is possible to exploit the proposed blending method to resolve locally the topological difficulties of an otherwise predominantly isogeometric approximation.

\section{Acknowledgements}

We gratefully acknowledge Hennadiy Netuzhylov and David Modesto for their technical support and invaluable discussions about isogeometric analysis and NEFEM methods, respectively. We acknowledge the support of the European Research Council un der the European Community's 7th Framework Programme (FP7/ 007 2013)/ERC Grant agreement nr 240487 and the Ministerio de 


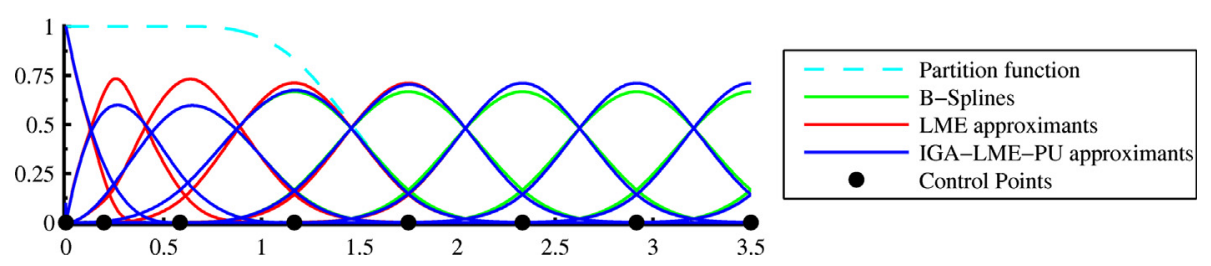

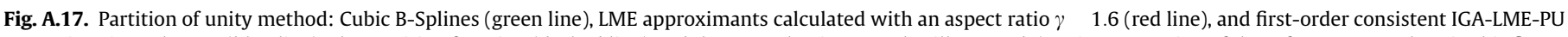

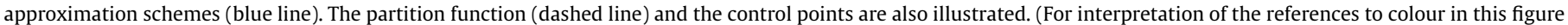
legend, the reader is referred to the web version of this article.)

Ciencia e Innovación (DPI2007 61054). MA acknowledges the sup port received through the prize "ICREA Academia" for excellence in research, funded by the Generalitat de Catalunya.

\section{Appendix A. Alternative methods to blend LME approximants and B-Splines}

We summarize in 1D the ideas behind two alternative strate gies to blend LME approximants and B Splines: (i) the partition of unity method, and (ii) the maximization of the relative entropy with B Splines and Shepard approximants as prior basis functions.

\section{A.1. Partition of unity method}

The partition of unity (PU) is a classical technique to design con forming spaces with specific properties [31]. This method enables us to glue together convex LME approximants and B Splines, de noted as $p_{a}(x)$ and $N_{a}(x)$ respectively, through the equation

$m_{a}(x) \quad[1 \quad \rho(x)] p_{a}(x)+\rho(x) N_{a}(x)$,

where $m_{a}(x)$ represents the new IGA LME PU approximation scheme, and $\rho(x) \in[0,1]$ is the function of partition. By construc tion, these approximants satisfy the conditions $m_{a}(x) \geqslant 0$ and $\sum_{a}^{N}{ }_{1} m_{a}(x)$ 1. The fulfillment of the first order reproducibility condition $\sum_{a}^{N} m_{a}(x) x_{a} \quad x$ is straightforward for an order $p \geqslant 1$. As the continuity of $m_{a}(x)$ is determined by the order of the B Splines (LME approximants are $C^{\infty}$ ), the function $\rho(x)$ has to be at least as continuous as the B Spline interpolants.

In Fig. A.17 we illustrate first order consistent IGA LME PU approximants for a one dimensional grid of points. The cubic $B$ Splines and the LME approximants $(\gamma \quad 1.6)$ used for the calcula tions are also depicted. The partition function is constructed by summing the first four cubic B Spline basis functions. Note that the new scheme is different to LME approximants only in the re gion of influence of the partition function. While this approach is very simple conceptually, the construction of the partition function and the B Spline basis functions requires a larger structured grid of points next to the boundary.

\section{A.2. Relative entropy maximization}

LME approximation schemes can be also derived from the maximization of the relative entropy $[29,32]$. The formalism is based on the Kullback Leibler distance [39], or negative relative entropy, between two discrete probability distributions $M$ and $R$

$D_{M / R} \quad \sum_{a}^{N} m_{a} \ln \frac{m_{a}}{r_{a}}$,

where $M \quad\left\{m_{a}\right\}_{a \quad 1, \ldots, N}, R \quad\left\{r_{a}\right\}_{a}{ }_{1 \ldots . N}$ are non negative numbers satisfying $\sum_{a}^{N} m_{a} \quad 1$, and $\sum_{a{ }_{1}}^{N} r_{a} \quad 1$. Notice that $D_{M / R}$ is not strictly a distance since it is not symmetric in its arguments. None theless, it is useful to think of as a measure of the "distance" be tween two distributions, and it is often interpreted as the amount of information needed to change the description of the system from $R$ to $M$. The probability distribution $R$ is often referred to as prior. A question in statistical inference is how to determine a new distribu tion $M$ as close as possible to some prior distribution $R$, but incorpo rating additional information in the form of constraints. A relative entropy maximization program provides a means to find such distribution:

$$
\begin{aligned}
\text { (RE) For fixed } x \text { minimize } & \sum_{a 1}^{N} m_{a} \ln \frac{m_{a}}{r_{a}(x)}, \\
\text { subject to } & m_{a} \geqslant 0, \sum_{a 1}^{N} m_{a} \quad 1, \sum_{a 1}^{N} m_{a} x_{a} \quad x .
\end{aligned}
$$

The solution of this problem can be written as $m_{a}(x) \quad r_{a}(x) \exp \left[f_{a}^{*}(x)\right] / Z_{r}(x)$, where the relative partition function is $Z_{r}(x) \quad \sum_{b{ }_{1}}^{N} r_{b}(x) \exp \left[f_{b}^{*}(x)\right]$, while $f_{a}^{*}(x)$ depends on the consis tency conditions. Notice that the resulting distributions are the product of the prior functions and a correction accounting for the extra constraints. These distributions can be understood as basis functions [1] and, for instance, LME approximation schemes are recovered when Shepard approximants with Gaussian kernel are used as prior probability distributions [32].

To blend max ent and B Splines, we consider B Splines and Shepard basis functions with Gaussian kernel as prior information in the relative entropy program. In Fig. A.18 we show the prior $\mathrm{cu}$ bic B Splines (four control points nearest to the boundary), the prior Shepard approximants (remaining points) for $\gamma 1.6$, and the resulting approximants, referred as IGA LME RE. Notice that these new approximation schemes are as smooth as the priors, and remain very close to the priors. If the priors satisfy the constraints, e.g. in the region close to the boundary, the resulting approximants coincide with the priors. For these reason, the IGA LME RE approximants follow the B Splines in the vicinity of the

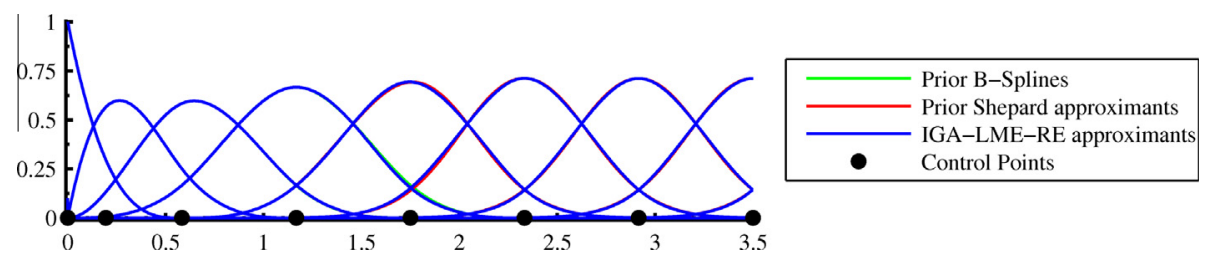

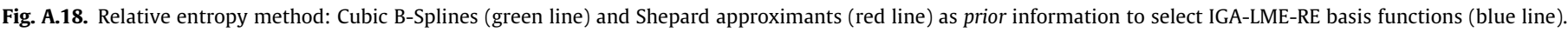
The control points are also illustrated. (For interpretation of the references to colour in this figure legend, the reader is referred to the web version of this article.) 
boundary, and mend the Shepard approximants to fulfill the consistency conditions. The behavior in the blending region de pends considerably on the parameter $\gamma$. This strategy is computa tionally much more expensive than the other two blending approaches.

\section{Appendix B. Spatial derivatives of the basis functions}

We provide here details for the calculation of the first spatial derivatives for the blended isogeometric/max ent approximation schemes. We denote partial differentiation by $\partial$, spatial gradients of scalar functions by $\nabla$, and the matrix of partial derivatives for vector valued functions by $D \boldsymbol{y}(\boldsymbol{x})$. The symbol ${ }^{*}$ is used to indicate that a function is evaluated at the optimal Lagrange multiplier $\lambda^{*}(\boldsymbol{x})$, which introduces explicit and implicit dependences on $\boldsymbol{x}$ in all functions with *. Within the scope of this appendix, we use $m_{a}^{*}$ to denote what has been referred to as $m_{a}$ in the rest of the pa per. No implied sum is assumed for repeated node indices.

As shown in Section 4, the optimal Lagrange multiplier $\lambda^{*}$ min imize the dual function $g(\boldsymbol{x}, \lambda) \quad A(\boldsymbol{x}) \ln Z(\boldsymbol{x}, \lambda)$, that is, $\lambda^{*}(\boldsymbol{x}) \quad \arg \min _{\lambda \in \mathbb{R}^{d}}\{g(\boldsymbol{x}, \lambda)\}$. We solve this nonlinear problem with the Newton Raphson method, which requires the calculation of the first and second derivatives of the objective function with re spect to the Lagrange multiplier

$$
\begin{array}{rll}
\boldsymbol{r}(\boldsymbol{x}, \lambda) & g_{\lambda}(\boldsymbol{x}, \lambda) & \sum_{a \in \mathscr{I}_{M E}} m_{a}(\boldsymbol{x}, \lambda)\left[\begin{array}{ll}
\boldsymbol{x} & \boldsymbol{x}_{a}+\frac{\boldsymbol{Y}(\boldsymbol{x})}{A(\boldsymbol{x})}
\end{array}\right], \\
\boldsymbol{J}(\boldsymbol{x}, \lambda) & g_{\lambda \lambda}(\boldsymbol{x}, \lambda) & \sum_{a \in \mathscr{I}_{M E}} m_{a}(\boldsymbol{x}, \lambda)\left[\begin{array}{ll}
\boldsymbol{x} & \boldsymbol{x}_{a}+\frac{\boldsymbol{Y}(\boldsymbol{x})}{A(\boldsymbol{x})}
\end{array}\right] \otimes\left[\begin{array}{ll}
\boldsymbol{x} & \boldsymbol{x}_{a}+\frac{\boldsymbol{Y}(\boldsymbol{x})}{A(\boldsymbol{x})}
\end{array}\right] \\
& \frac{1}{A(\boldsymbol{x})} \boldsymbol{r}(\boldsymbol{x}, \lambda) \otimes \boldsymbol{r}(\boldsymbol{x}, \lambda) .
\end{array}
$$

Hereafter, the dependence on the variables $\boldsymbol{x}$ and $\boldsymbol{\lambda}$ will be dropped in order to simplify the notation. By taking the spatial derivatives of the expression $m_{a}^{*} \quad \exp \left(f_{a}^{*}\right) / Z^{*}$ (see Section 4), it is readily verified that

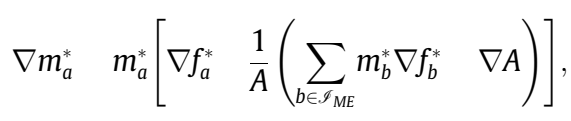

where $\nabla A \quad \sum_{b \in \mathscr{I}_{B S}} \nabla N_{b}$. Applying the chain rule to the equation

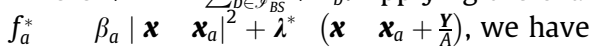

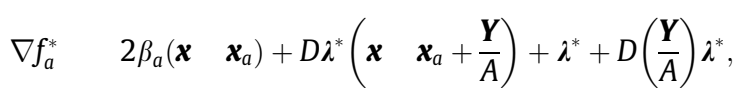

where

$$
\begin{aligned}
& D\left(\frac{\boldsymbol{Y}}{A}\right) \quad \frac{1}{A} D \boldsymbol{Y} \quad \frac{1}{A^{2}} \nabla A \otimes \boldsymbol{Y} \\
& \frac{1}{A} \sum_{b \in \mathscr{I}_{B S}} \nabla N_{b} \otimes\left(\boldsymbol{x} \quad \boldsymbol{x}_{b}\right)+\frac{1 \quad A}{A} \boldsymbol{I d} \quad \frac{1}{A^{2}} \nabla A \otimes \boldsymbol{Y}
\end{aligned}
$$

and Id represents the identity matrix. By replacing the expression of $\nabla f_{a}^{*}$ in Eq. (B.1) and introducing

$$
\boldsymbol{r}_{\beta} \quad 2 \sum_{a \in \mathscr{I}_{M E}} \beta_{a} m_{a}^{*}\left(\boldsymbol{x} \quad \boldsymbol{x}_{a}\right),
$$

we obtain

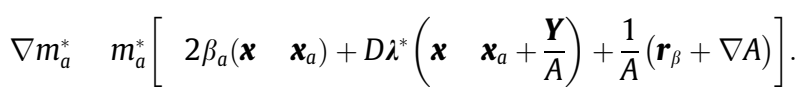

The term $D \lambda^{*}$ is not explicitly available but, given that $\boldsymbol{r}^{*}$ is identi cally zero (first order reproducibility condition), the following con dition is also satisfied $\mathbf{0} \quad D \boldsymbol{r}^{*} \quad \boldsymbol{J}_{\beta}+A \boldsymbol{I d}+A D\left(\frac{\boldsymbol{Y}}{A}\right)+D \lambda^{*} \boldsymbol{J}^{*}$

where

$\boldsymbol{J}_{\beta} \quad 2 \sum_{a \in \mathscr{I}_{M E}} \beta_{a} m_{a}^{*}\left(\boldsymbol{x} \quad \boldsymbol{x}_{a}\right) \otimes\left(\begin{array}{ll}\boldsymbol{x} & \left.\boldsymbol{x}_{a}+\frac{\boldsymbol{Y}}{A}\right) .\end{array}\right.$

From the previous equations it follows that

$D \lambda^{*} \quad\left[\boldsymbol{J}_{\beta} \quad A \boldsymbol{I d} \quad A D\left(\frac{\boldsymbol{Y}}{A}\right)\right]\left(\boldsymbol{J}^{*}\right)^{1}$,

which leads to a close expression for the first spatial derivatives of the basis functions

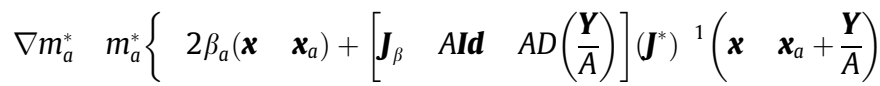

$$
\begin{aligned}
& \left.+\frac{1}{A}\left(\boldsymbol{r}_{\beta}+\nabla A\right)\right\} \text {. }
\end{aligned}
$$

Note that for the points where $A \quad$ 1, and consequently $\boldsymbol{Y} \quad \mathbf{0}$, we recover the expression provided in [9], that is

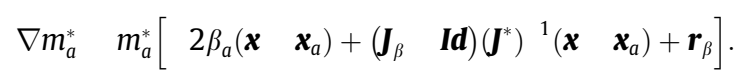

\section{References}

[1] M. Arroyo, M. Ortiz, Local maximum-entropy approximation schemes: a seamless bridge between finite elements and meshfree methods, Int. J. Numer. Methods Engrg. 65 (13) (2006) 2167-2202.

[2] N. Sukumar, Construction of polygonal interpolants: a maximum entropy approach, Int. J. Numer. Methods Engrg. 61 (12) (2004) 2159-2181.

[3] N. Sukumar, B. Moran, T. Belytschko, The natural element method in solid mechanics, Int. J. Numer. Methods Engrg. 43 (5) (1998) 839-887.

[4] F. Cirak, M. Ortiz, P. Schröder, Subdivision surfaces: a new paradigm for thinshell finite-element analysis, Int. J. Numer. Methods Engrg. 47 (12) (2000) 2039-2072.

[5] T. Hughes, J. Cottrell, Y. Bazilevs, Isogeometric analysis: CAD, finite elements, NURBS, exact geometry and mesh refinement, Comput. Methods Appl. Mech. Engrg. 194 (2005) 4135-4195.

[6] C. Cyron, M. Arroyo, M. Ortiz, Smooth, second order, non-negative meshfree approximants selected by maximum entropy, Int. J. Numer. Methods Engrg. 79 (13) (2009) 1605-1632.

[7] A. Rosolen, D. Millán, M. Arroyo, Second order convex maximum entropy approximants with applications to high order PDE, Int. J. Numer. Methods Engrg. 94 (2) (2013) 150-182.

[8] A. Bompadre, L.E. Perotti, C. Cyron, M. Ortiz, Convergent meshfree approximation schemes of arbitrary order and smoothness, Comput. Methods Appl. Mech. Engrg. 221-222 (2012) 83-103.

[9] A. Rosolen, D. Millán, M. Arroyo, On the optimum support size in meshfree methods: a variational adaptivity approach with maximum entropy approximants, Int. J. Numer. Methods Engrg. 82 (7) (2010) 868-895.

[10] S. Fernández-Méndez, A. Huerta, Imposing essential boundary conditions in mesh-free methods, Comput. Methods Appl. Mech. Engrg. 193 (12-14) (2004) 1257-1275.

[11] H. Edelsbrunner, E. Mücke, Three-dimensional alpha shapes, ACM Trans. Graphics 13 (1) (1994) 43-72.

[12] J. Cottrell, T. Hughes, Y. Bazilevs, Isogeometric Analysis: Toward Integration of CAD and FEA, John Wiley \& Sons, Ltd., 2009.

[13] Y. Bazilevs, V. Calo, J. Cottrell, J. Evans, T. Hughes, S. Lipton, M. Scott, T. Sederberg, Isogeometric analysis using T-splines, Comput. Methods Appl. Mech. Engrg. 199 (5-8) (2010) 229-263.

[14] L.B. da Veiga, A. Buffa, D. Cho, G. Sangalli, Isogeometric analysis using T-splines on two-patch geometries, Comput. Methods Appl. Mech. Engrg. 200 (21-22) (2011) 1787-1803.

[15] M. Dörfel, B. Jüttler, B. Simeon, Adaptive isogeometric analysis by local hrefinement with T-splines, Comput. Methods Appl. Mech. Engrg. 199 (5-8) (2010) 264-275.

[16] N. Nguyen-Thanh, H. Nguyen-Xuan, S. Bordas, T. Rabczuk, Isogeometric analysis using polynomial splines over hierarchical T-meshes for twodimensional elastic solids, Comput. Methods Appl. Mech. Engrg. 200 (21-22) (2011) 1892-1908.

[17] M. Scott, X. Li, T. Sederberg, T. Hughes, Local refinement of analysis-suitable Tsplines, Comput. Methods Appl. Mech. Engrg. 213-216 (2012) 206-222.

[18] A.-V. Vuong, C. Giannelli, B. Jüttler, B. Simeon, A hierarchical approach to adaptive local refinement in isogeometric analysis, Comput. Methods Appl. Mech. Engrg. 200 (49-52) (2011) 3554-3567. 
[19] H.-J. Kim, Y.-D. Seo, S.-K. Youn, Isogeometric analysis with trimming technique for problems of arbitrary complex topology, Comput. Methods Appl. Mech. Engrg. 199 (45-48) (2010) 2796-2812.

[20] D. Schillinger, L. Dedè, M.A. Scott, J.A. Evans, M.J. Borden, E. Rank, T.J. Hughes, An isogeometric design-through-analysis methodology based on adaptive hierarchical refinement of nurbs, immersed boundary methods, and T-Spline cad surfaces, Comput. Methods Appl. Mech. Engrg. 249-252 (2012) 116-150.

[21] E. Rank, M. Ruess, S. Kollmannsberger, D. Schillinger, A. Düster, Geometric modeling, isogeometric analysis and the finite cell method, Comput. Methods Appl. Mech. Engrg. 249-252 (2012) 104-115.

[22] D. Burkhart, B. Hamann, G. Umlauf, Iso-geometric finite element analysis based on Catmull-Clark subdivision solids, Comput. Graphics Forum 29 (5) (2010) 1575-1584.

[23] A. Rosolen, C. Peco, M. Arroyo, An adaptive meshfree method for phase-field models of biomembranes. Part I: Approximation with maximum-entropy approximants, J. Comput. Phys. (2013), http://dx.doi.org/10.1016/ j.jcp.2013.04.046.

[24] D. Millán, A. Rosolen, M. Arroyo, Nonlinear manifold learning for meshfree finite deformation thin shell analysis, Int. J. Numer. Methods Engrg. 93 (7) (2013) 685-713.

[25] D. Millán, A. Rosolen, M. Arroyo, Thin shell analysis from scattered points with maximum-entropy approximants, Int. J. Numer. Methods Engrg. 85 (6) (2011) 723-751.

[26] R. Sevilla, S. Fernández-Méndez, A. Huerta, 3D NURBS-enhanced finite element method (NEFEM), Int. J. Numer. Methods Engrg. 88 (2) (2011) 103-125.

[27] A. Huerta, S. Fernández-Méndez, Enrichment and coupling of the finite element and meshless methods, Int. J. Numer. Methods Engrg. 48 (2000) 1615-1636
[28] D. González, E. Cueto, M. Doblaré, A higher-order method based on local maximum entropy approximation, Int. J. Numer. Methods Engrg. 83 (6) (2010) 741-764.

[29] N. Sukumar, R. Wright, Overview and construction of meshfree basis functions: from moving least squares to entropy approximants, Int. J. Numer. Methods Engrg. 70 (2) (2007) 181-205.

[30] L. Piegl, W. Tiller, The NURBS Book, Springer, 1997.

[31] I. Babuska, J. Melenk, The partition of unity method, Int. J. Numer. Methods Engrg. 40 (1996) 727-758.

[32] M. Arroyo, M. Ortiz, Local maximum-entropy approximation schemes, Meshfree Methods for Partial Differential Equations III, Lecture Notes in Computational Science and Engineering, vol. 57, Springer, 2007. pp. 116.

[33] X. Roca, E. Ruiz-Gironés, J. Sarrate, A graphical modeling and mesh generation environment for simulations based on boundary representation data, Congresso de métodos numéricos em engenharia, Porto, Portugal, 2007.

[34] X. Roca, Paving the path towards automatic hexahedral mesh generation, Ph.D. thesis, Universitat Politècnica de Catalunya, 2009.

[35] X. Roca, E. Ruiz-Gironés, J. Sarrate, ez4u mesh generation environment, 2010. $<$ http://www-lacan.upc.edu/ez4u.htm>.

[36] R. Sevilla, S. Fernández-Méndez, A. Huerta, Comparison of high-order curved finite elements, Int. J. Numer. Methods Engrg. 87 (8) (2011) 719-734.

[37] D. Salomon, Curves and Surfaces for Computer Graphics, Springer, 2006

[38] S. Timoshenko, J. Goodier, Theory of Elasticity, McGraw-Hill, New York, 1951.

[39] S. Kullback, R. Leibler, On information and sufficiency, Ann. Math. Stat. 22 (1951) 79-86. 